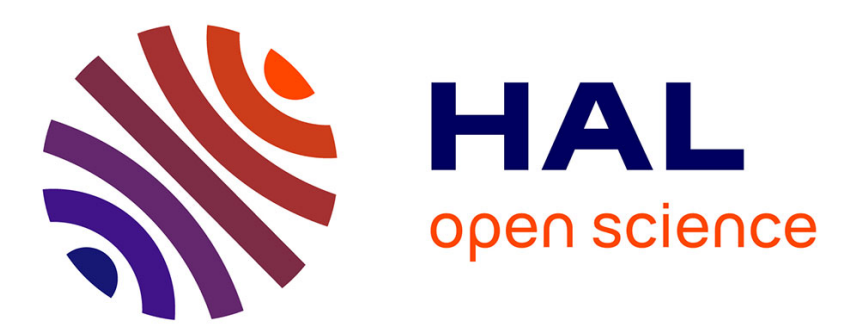

\title{
Analysis of a Generalized Dispersive Model Coupled to a DGTD Method with Application to Nanophotonics
}

\author{
Stephane Lanteri, Claire Scheid, Jonathan Viquerat
}

\section{To cite this version:}

Stephane Lanteri, Claire Scheid, Jonathan Viquerat. Analysis of a Generalized Dispersive Model Coupled to a DGTD Method with Application to Nanophotonics. SIAM Journal on Scientific Computing, 2017, 39 (3), pp.A831 - A859. 10.1137/15M105207X . hal-01674031

\section{HAL Id: hal-01674031 \\ https://hal.inria.fr/hal-01674031}

Submitted on 10 Jan 2018

HAL is a multi-disciplinary open access archive for the deposit and dissemination of scientific research documents, whether they are published or not. The documents may come from teaching and research institutions in France or abroad, or from public or private research centers.
L'archive ouverte pluridisciplinaire HAL, est destinée au dépôt et à la diffusion de documents scientifiques de niveau recherche, publiés ou non, émanant des établissements d'enseignement et de recherche français ou étrangers, des laboratoires publics ou privés. 


\title{
ANALYSIS OF A GENERALIZED DISPERSIVE MODEL COUPLED TO A DGTD METHOD WITH APPLICATION TO NANOPHOTONICS
}

\author{
S. LANTERI, C. SCHEID, J. VIQUERAT*
}

\begin{abstract}
In this paper, we are concerned with the numerical modelling of the propagation of electromagnetic waves in dispersive materials for nanophotonics applications. We focus on a generalized model that allows for the description of a wide range of dispersive media. The underlying differential equations are recast into a generic form and we establish an existence and uniqueness result. We then turn to the numerical treatment and propose an appropriate Discontinuous Galerkin Time Domain framework. We obtain the semi-discrete convergence and prove the stability (and in a larger extent, convergence) of a Runge Kutta 4 fully discrete scheme via a technique relying on energy principles. Finally, we validate our approach through two significant nanophotonics test cases.
\end{abstract}

Key words. Nanophotonics, Maxwell's equations, dispersive media, Discontinuous Galerkin, Runge Kutta schemes.

AMS subject classifications. 97N40, 35Q61, 65Z05

1. Introduction. Among the numerous phenomena encountered in electromagnetics, many rely on the dispersive properties of materials, e.g. the fact that their phase velocity varies with frequency. Indeed, in specific ranges of wavelengths, biological tissues GGC96, noble JC72 and transition metals JC74, but also glass Fle78 and certain polymers [CC41] exhibit non-negligible dispersive behaviors. From the mathematical modeling point of view, this phenomenon is modeled by a frequencydependent permittivity function $\varepsilon(\omega)$, often derived from physical considerations. Regarding nanophotonics applications, an accurate modeling of the permittivity function for metals in the visible spectrum is crucial. Indeed, the free electrons of metals are the key ingredient in the propagation of surface modes of particular interest, called surface plasmons $\mathrm{NH} 07$.

The implementation of dispersion models in time-domain electromagnetics solvers can be achieved by different methods. The most common is certainly the Additional Differential Equation (ADE) technique, which consists in the addition of one or more ODEs to the Maxwell system, the coupling being made via source terms. A consequent literature on this topic exists in the context of Finite-Difference Time-Domain (FDTD) (see e.g. [VLDC11] and references therein). More recently, more papers are concerned with Finite Element or even Discontinuous Galerkin Time-Domain approaches (DGTD) (see e.g. GYKR12] and [BKN11] and references therein), aiming at overcoming the limitations of FDTD. In this context, some works are more precisely focused on the numerical analysis. Several proofs exist for the standard dispersive media models and the most classical time and space discretization schemes (see e.g. all the papers of J. Li and co-authors such as [JL06, Li07, LCE08, Li09]). Let us also mention the approach of [WXZ10 for the integro-differential version of the classical dispersive models. The latter reference propose to analyze a semi-discrete divergence free discontinuous Galerkin framework. Finally, in a previous work [LS13, the authors analyzed, for the Debye model, a fully discrete scheme based on a centered fluxes nodal Discontinuous Galerkin formulation and Leap frog discretization in time.

In this paper, we present a complete study of a generalized dispersive model that encapsulates a wide range of dispersive media, its higher efficiency being demonstrated

*The PhD thesis of J. Viquerat is partially funded by the DGA 
when compared with standard models for dispersive metals (i.e. Drude and Drude Lorentz models). After having proven the existence and uniqueness of the solution, we propose an implementation of the target model in a DGTD framework, for which stability and convergence of the semi-discrete scheme are established. We examine a formulation allowing for center fluxes to fully upwind fluxes. A Runge-Kutta 4 (RK4) time-integration scheme is then introduced, and stability is established using energy principles. It extends the approach of [BEF10] that was valid for lower order Runge Kutta time schemes. The convergence result is quickly sketched since arguments then closely follow BEF10, LS13. We end this study with two tridimensional numerical tests related to nanophotonics: (i) the calculation of the reflection coefficient of an infinite dispersive slab which is used to confirm the good implementation of our method, and (ii) the absorption cross-section calculation of a gold-silver nano-shell to demonstrate the importance of a good description of the dispersive properties in metals for nanophotonics-related computations.

2. Dispersion models. In this section, we first introduce Maxwell's equations and the most classical dispersion models used for metals in nanophotonics. Then, the Generalized Dispersive model (GD) is introduced, and its advantages over classical models are presented. Eventually, the causality principle is recalled, and a causality condition is obtained for the GD model.

2.1. Maxwell's equation in dispersive media. Let us present, formally, the modelling of the propagation of electromagnetic waves in dispersive media. It relies on the set of Maxwell's equations:

$$
\left\{\begin{array}{l}
\frac{\partial \mathbf{B}}{\partial t}=-\nabla \times \mathbf{E}+\mathbf{J} \\
\frac{\partial \mathbf{D}}{\partial t}=\nabla \times \mathbf{H}
\end{array}\right.
$$

with $\mathbf{J}$ a current source term. In addition to boundary (or radiation) condition and initial conditions, constitutive relations are added to this system of equations. They link the electric displacement $\mathbf{D}$ and the electric field $\mathbf{E}$ and the magnetic induction $\mathbf{B}$ and the magnetic field $\mathbf{H}$. In the sequel, we consider non magnetic linear homogeneous material, so that:

$$
\mathbf{B}=\mu_{0} \mathbf{H},
$$

with $\mu_{0}$ the vacuum permeability. The electric constitutive relation in a linear, homogeneous, non dispersive material is given by a proportionality relation between $\mathbf{D}$ and $\mathbf{E}$ as:

$$
\mathbf{D}=\varepsilon_{0} \varepsilon_{r} \mathbf{E},
$$

with $\varepsilon_{0}$ the vacuum permittivity and $\varepsilon_{r}$ the constant relative permittivity describing the electric reaction of the medium. To enlighten the approach that follows in the next two subsections, we focus, for a moment, on the electric constitutive relation in the frequency domain. To this end, we suppose a harmonic time dependence on the fields and consider the Fourier transform of the involved fields at a given frequency $\omega$. In this setting the dispersive character of a medium is expressed as a frequency dependent and complex valued permittivity $\hat{\varepsilon}_{r}(\omega)$ in the constitutive relation. Here the notation $\hat{\imath}$ stands for the Fourier transform of the corresponding field. Thus, if 


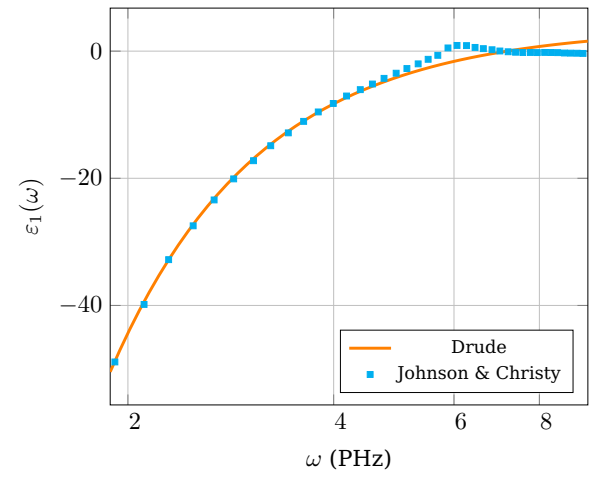

(a) Real part

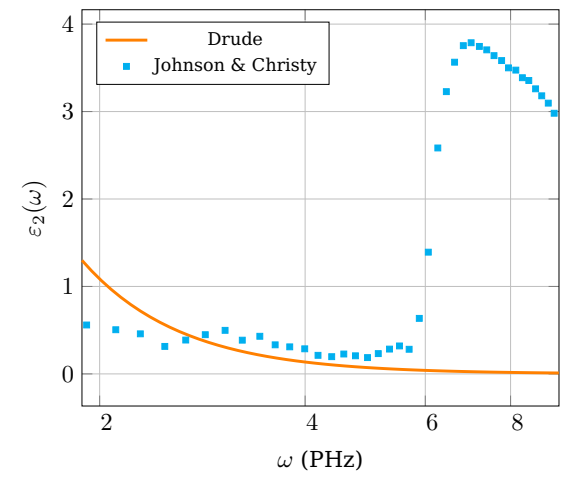

(b) Imaginary part

FIG. 1. Real and imaginary parts of the silver relative permittivity predicted by the Drude model compared to experimental data from Johnson $\&$ Christy. The parameter values are $\varepsilon_{\infty}=$ 3.7362, $\omega_{d}=1.3871 \times 10^{7} \mathrm{GHz}$ and $\gamma_{d}=4.5154 \times 10^{4} \mathrm{GHz}$.

one wants to prescribe a dispersion model, one possible choice consists in determining an appropriate complex-valued function $\hat{\varepsilon}_{r}$ according to physical and/or experimental considerations. Each expression of $\hat{\varepsilon}_{r}$ will thus lead to a different time domain model, by simply performing an inverse Fourier transform.

2.2. Drude and Lorentz models. The Drude model is based on the kinetic theory of gases Dru00. In this approximation, the metal is considered as a static lattice of positive ions immersed in a free electrons gas. The interactions of these electrons with the ion lattice are materialized by a collision frequency parameter $\gamma_{d}$. For a single electron, this leads to the following classical equation of motion for the speed $\mathbf{v}$ :

$$
\frac{\partial \mathbf{v}}{\partial t}+\gamma_{d} \mathbf{v}=-\frac{e}{m_{e}} \mathbf{E}(t)
$$

where $m_{e}$ represents the electron mass, and $e$ the electronic charge. One could notice that $\left[\gamma_{d}\right]=T^{-1}$, and $\gamma_{d}$ matches the definition of the inverse of the mean free path denoted by $\tau_{f}$. Then, considering a harmonic time-dependence, using Ohm's law, one extracts the expression of the frequency dependent conductivity that permits to express the permittivity. A few manipulations yield the expression of the permittivity in terms of the frequency $\omega$ (the interested reader is referred to [LSV13] for the detailed steps) 1 .

$$
\hat{\varepsilon}_{r, d}(\omega)=\varepsilon_{\infty}-\frac{\omega_{d}^{2}}{\omega^{2}+i \omega \gamma_{d}}
$$

where $\omega_{d}=\sqrt{\frac{n_{e} e^{2}}{m_{e} \varepsilon_{0}}}$ is the plasma frequency of the electrons, with $n_{e}$ the electronic density and $\varepsilon_{\infty}$ is the permittivity at infinite frequency. The real and imaginary parts of the Drude permittivity function of the silver are plotted in figure 1, along with experimental data from Johnson and Christy [JC72].

\footnotetext{
${ }^{1}$ The subscript $d$ stands for Drude.
} 


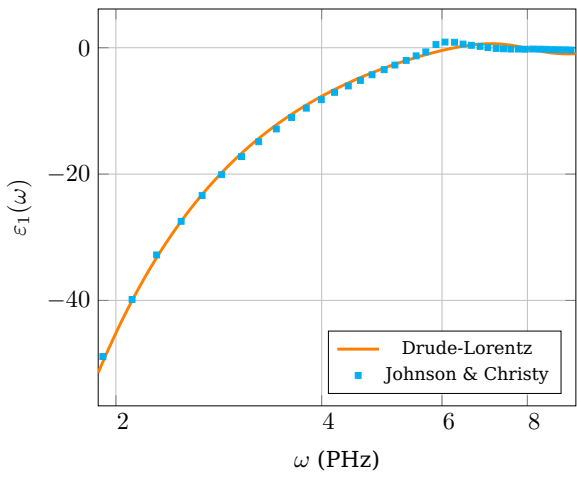

(a) Real part

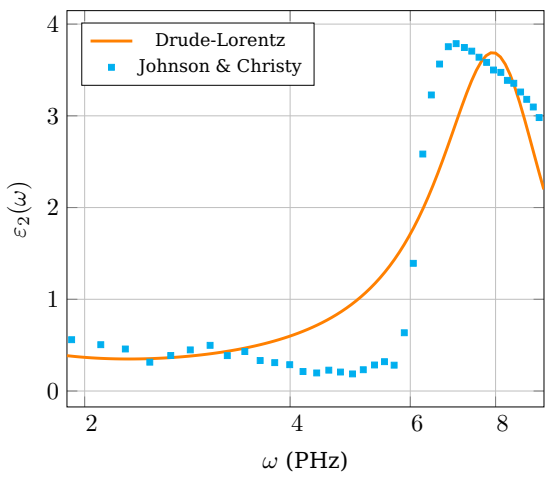

(b) Imaginary part

FIG. 2. Real and imaginary parts of the silver relative permittivity predicted by the DrudeLorentz model compared to experimental data from Johnson 85 Christy. The parameter values are $\varepsilon_{\infty}=2.7311, \omega_{d}=1.4084 \times 10^{7} \mathrm{GHz}, \gamma_{d}=6.6786 \times 10^{3} \mathrm{GHz}, \Delta \varepsilon=1.6336, \omega_{l}=8.1286 \times 10^{6}$ GHz and $\gamma_{l}=3.6448 \times 10^{6} \mathrm{GHz}$.

One should notice that, if the real part is fitted by the Drude prediction, the experimental imaginary part shows features that are not predicted by the model. For certain metals (especially noble ones), electronic transitions between valence and conduction band occur around the visible frequency range. These contributions correspond to electrons that are bound to their ion cores. Hence, in the same classical fashion as before, a spring term is added to the equation of motion with resonant frequency $\omega_{l}$ and damping $\gamma_{l}$ :

$$
\frac{\partial^{2} \mathbf{x}}{\partial t^{2}}+\gamma_{l} \frac{\partial \mathbf{x}}{\partial t}+\omega_{l}^{2} \mathbf{x}=-\frac{e}{m_{e}} \mathbf{E}(t)
$$

where $x$ denotes the position of the electrons.

This is the Lorentz model. Following the same development as for the Drude model, one easily obtains the expression of a Lorentz pole:

$$
\hat{\varepsilon}_{r, l}(\omega)=-\frac{\Delta \varepsilon \omega_{l}^{2}}{\omega^{2}-\omega_{l}^{2}+i \omega \gamma_{l}},
$$

with $\Delta \varepsilon$ a constant related to the pole strength.

The total permittivity of the Drude-Lorentz model is the simple addition of the Drude and Lorentz terms:

$$
\hat{\varepsilon}_{r, d l}(\omega)=\varepsilon_{\infty}-\frac{\omega_{d}^{2}}{\omega^{2}+i \omega \gamma_{d}}-\frac{\Delta \varepsilon \omega_{l}^{2}}{\omega^{2}-\omega_{l}^{2}+i \omega \gamma_{l}} .
$$

As can be seen for silver in figure 2, the high-frequency range of the imaginary part is in better agreement with experimental data than it was for the Drude model. However, there is still room for improvement: for some metals such as gold or silver, the addition of multiple Lorentz terms brings a much better fit between experimental and theoretical values, at the cost of an increased complexity of the model. Based on this remark, the L4 model [HN07 combines four Lorentz poles with a conductivity term. 
2.3. Generalized dispersive model. Given an experimental set of points describing a permittivity function of a material, a Padé type approximation could be a convenient analytical coefficient-based function to approach experimental data. The fundamental theorem of algebra then allows to expand this approximation as a sum of a constant, a set of first-order generalized poles (FOGP), and a set of second-order generalized poles (SOGP), respectively as:

$$
\hat{\varepsilon}_{r, g}(\omega)=\varepsilon_{\infty}-\frac{\sigma}{i \omega}-\sum_{l \in L_{1}} \frac{a_{l}}{i \omega-b_{l}}-\sum_{l \in L_{2}} \frac{c_{l}-i \omega d_{l}}{\omega^{2}-e_{l}+i \omega f_{l}},
$$

where $\varepsilon_{\infty}, \sigma, a_{l}, b_{l}, c_{l}, d_{l}, e_{l}, f_{l}$ are real constants, and $L_{1}, L_{2}$ are sets of indices. The constant $\varepsilon_{\infty}$ represents the permittivity at infinite frequency, and $\sigma$ the constant conductivity.

This general writing allows an important flexibility for several reasons. First, it unifies most of the common dispersion models in a single formulation. Indeed, Debye (biological tissues in the $\mathrm{MHz}$ regime), Drude and Drude-Lorentz (noble metals in the $\mathrm{THz}$ regime), retarded Drude and Drude-Lorentz (transition metals in the THz regime), but also Sellmeier's law (glass in the THz regime), are naturally included. Second, as will be shown later, it permits to fit a large range of experimental data set in a reasonable number of poles (thus leading to reasonable memory and CPU overheads). A similar approach was used in the case of the Critical Points (CP) model with two (see [VLDC11]) and three (see [LC09]) poles, and in the Complex-Conjugate PoleResidue Pairs model (CCPRP) (see [HDF06]). In essence, these techniques allow for complex coefficients in their developments, and can therefore yield a decomposition of the permittivity function in pairs of single-order poles only, whereas choosing real coefficients leads to a collection of first-order and second-order poles. However, the numerical complexities of their implementations is equivalent to the one of the GD model. In the remaining of this paper, we focus on SOGP only, since they are the most pertinent ones in nanophotonics applications.

Computation of the GD model coefficients. In order to fit the coefficients of (2.3) to experimental data, various techniques can be used, such as the well-known least square method. Vector fitting techniques (see GS99]) are also well developed for the CCPRP formulation. For an increasing number of poles, one can be left with a large optimization problem presenting many local maxima. Simulated Annealing (SA) methods have proved to be particularly efficient in finding global maxima in these situations, even when the initial guess is far from the optimal point ([KGV83). Hence, we adapted a free existing algorithm from Goffe 2 to the needs of this study. In practice, for a given model, a set of experimental data is provided to the optimization algorithm: in this study, we exploited the well-known Johnson and Christy tables ([JC72], [JC74]), although other are also widely-used [Pal98]. This method demonstrated good efficiency while fitting up to 17 parameters simultaneously.

Comparison with classical models. A key point in the quality of the fitting is the wideness of the spectrum of interest. Indeed, for a fixed number of parameters and poles, and depending on the behavior of the experimental permittivity function in the selected frequency range, one can obtain a good or a poor fit. In the remaining of this paper, the frequency interval is set to $[300,1500] \mathrm{THz}$, which constitutes a wide enough range for nanophotonics problems.

$\sqrt[2]{\text { http }: / / i d e a s . r e p e c . o r g / c / w p a / w u w p p r / 9406001 . h t m l ~}$ 
TABLE 1

Quality of the fit obtained by various dispersion models for gold and silver in the $[300,1500]$ THz range.

\begin{tabular}{clllll}
\hline & \multicolumn{2}{c}{ Silver } & & \multicolumn{2}{c}{ Gold } \\
\cline { 2 - 3 } \cline { 5 - 6 } & $\Delta_{r}$ & $\Delta_{i}$ & & $\Delta_{r}$ & $\Delta_{i}$ \\
\hline Drude & 0.8366 & 1.622 & & 1.715 & 3.752 \\
\hline Drude-Lorentz & 0.4649 & 0.4412 & & 0.5482 & 0.5759 \\
\hline L4 & 0.2028 & 0.2199 & & 0.2354 & 0.3256 \\
\hline 1SOGP & 0.8366 & 1.738 & 1.328 & 2.960 \\
\hline 2SOGP & 0.2061 & 0.2458 & & 0.2092 & 0.1843 \\
\hline 4SOGP & 0.08636 & 0.07786 & 0.09804 & 0.1083 \\
\hline
\end{tabular}

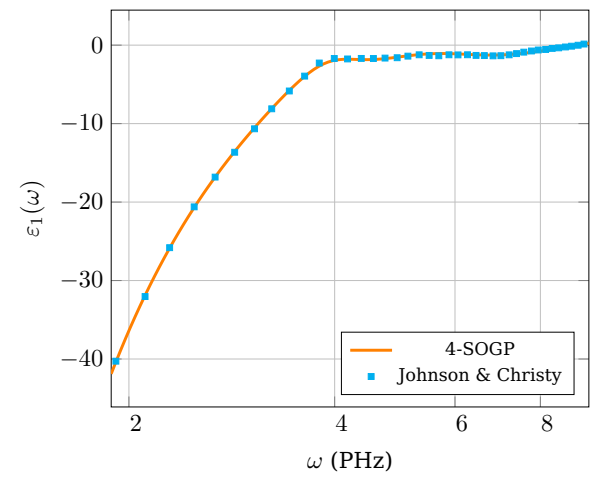

(a) Real part

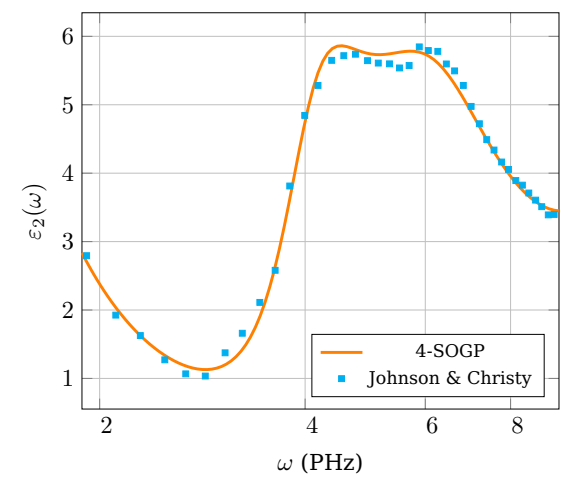

(b) Imaginary part

FIG. 3. Real and imaginary parts of the gold relative permittivity predicted by the 4 SOGP model compared to experimental data from Johnson 86 Christy. The parameter values can be found in [LSV13].

For gold and silver, we compare the quality of the results obtained with Drude, DrudeLorentz, 2SOGP, 4SOGP, and L4 models. All the parameters were fitted with the SA algorithm. The quality of the fit is evaluated by a point-by-point $L^{1}$ error normalized by the number of experimental samples. We note $\Delta_{r}$ the quality of the real part fit, and $\Delta_{i}$ the one of the imaginary part. Results are displayed in table 1. As can be seen, using SOGP instead of classical Drude and Lorentz poles provides a neat benefit in the description of the permittivity function. The 2SOGP and 4SOGP fit roughly and reduce the errors by a factor 2 when compared to the Drude-Lorentz and L4 fits, for both gold and silver. The only case where no improvement is obtained is the SOGP fit for silver, compared to the Drude model. As an illustration, the fitting obtained for gold with the 4SOGP model is presented in figure 3

A few words about transition metals. Although gold and silver are used for the most part, transition metals, such as nickel, cobalt or iron, were recently considered for plasmonic applications $\mathrm{PPM}^{+} 14$. However, the permittivity functions of such metals cannot be represented by the classical Drude model, since the latter assumes the electrons to be non-correlated. In transition metals, however, this is assumption is not true, and the global equilibrium is not reached instantaneously, inducing a 
TABLE 2

Quality of the fit obtained by Drude and 1SOGP models for nickel in the $[300,1500]$ THz range.

\begin{tabular}{ccc}
\hline & \multicolumn{2}{c}{ Nickel } \\
\cline { 2 - 3 } & $\Delta_{r}$ & $\Delta_{i}$ \\
\hline Drude & 1.079 & 8.323 \\
\hline 1SOGP & 1.1272 & 0.8750 \\
\hline
\end{tabular}

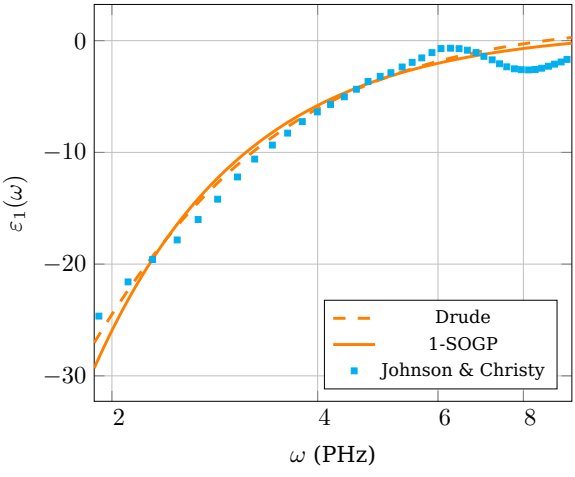

(a) Real part

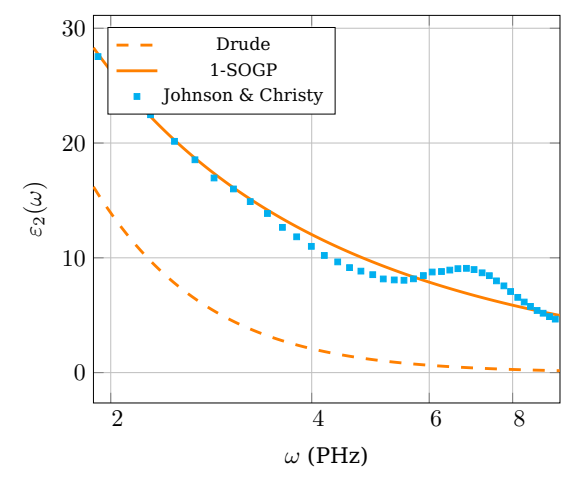

(b) Imaginary part

FIG. 4. Real and imaginary parts of the silver relative permittivity predicted by the Drude and the 1SOGP model compared to experimental data from Johnson 83 Christy. The parameters values are, for Drude: $\varepsilon_{\infty}=1.78, \omega_{d}=1.16 \times 10^{7} \mathrm{GHz}$ and $\gamma_{d}=1.058 \times 10^{6} \mathrm{GHz}$; for $1 \mathrm{SOGP}$ : $\varepsilon_{\infty}=1.0, c_{1}=1.1943 \times 10^{14} \mathrm{GHz}^{2}, d_{1}=4.6603 \times 10^{7} \mathrm{GHz}, e_{1}=0.0 \mathrm{GHz}^{2}$ and $f_{1}=2.2176 \times 10^{5}$ GHz.

retardation effect WROB13. In the latter reference, a retarded Drude model is derived from physical considerations, which can be represented by a proper choice of parameters with a SOGP, the $d_{l}$ parameter in 2.3 being linked to the relaxation time scale. Here, we illustrate this feature by simply computing coefficients for nickel with Drude and 1-SOGP models: the results are displayed in table 2, and a graphic representation can be found in figure 4. While the real part is close to experimental data, one clearly sees how the Drude model underestimates the losses in the metal. The improvement of the imaginary part with the 1-SOGP model is very appreciable, for a constant memory cost.

2.4. Causality principle. Whatever the chosen dispersion model, the latter has to respect the causality principle which relies on the natural observation that any physical system should not depend on future states of the system. This principle can be expressed mathematically thanks to the Kramers-Krönig relations as an analyticity condition for the frequency-dependent permittivity function. Even if this characterization is well established among physicists, the justification of this condition may sometimes be quite unclear. Hence, we found interesting to recall here the origin of this condition in a more mathematical framework.

The starting point is the fundamental relation between the electric displacement $\mathbf{D}$, the permittivity $\varepsilon$, and the electric field $\mathbf{E}$, namely $\mathbf{D}=\varepsilon * \mathbf{E}$.

This is a space-time convolution. However, since we are not focusing on any nonlocality in space but rather the nonlocality in time, in terms of dispersion effects, we 
will restrict our attention to the time convolution.

In other words,

$$
\mathbf{D}(t)=\int_{-\infty}^{\infty} \varepsilon\left(t-t^{\prime}\right) \mathbf{E}\left(t^{\prime}\right) d t^{\prime}
$$

This means that in all generality, the value of $\mathbf{D}$ may depend on future values of E. This contradicts the causality principle, if one does not impose any additional constraints to $\varepsilon$. The causality principle is then ensured by assuming that:

$$
\varepsilon(t)=0, \forall t<0 .
$$

Under the condition (2.4), we will say that the physical problem defined by $\varepsilon$ is causal.

Let us recall the following theorem stated in [Tit48] (theorem 95, p. 128).

TheOREM 2.1. Let $\phi \in L^{2}(\mathbb{R})$ be a complex valued function. The following conditions are equivalent:

(a) $\phi$ is a limit as $z=x+i y \rightarrow x$ of an analytic function $\Phi$ in the upper half plane (i.e. $y>0$ ) such that $\int_{-\infty}^{+\infty}|\Phi(x+i y)|^{2} d x<K$.

(b) $\phi(x)=f(x)-i g(x)$, where $f$ and $g$ are conjugate functions of $L^{2}$ (in the sense of Hilbert transforms, see [Tit48]).

(c) $\varphi$, the inverse Fourier transform of $\phi$, is such that $\varphi(x)=0, \forall x<0$.

A straightforward application of this theorem will give the conditions of causality.

THEOREM 2.2 (Causality principle).

Let $\varepsilon \in \varepsilon_{\infty} \delta+L^{2}(\mathbb{R})$ be a real valued function and $\hat{\varepsilon} \in \varepsilon_{\infty}+L^{2}(\mathbb{R})$ be the complex valued function defined as the Fourier transform of $\varepsilon$. The three following conditions (i), (ii) and (iii) are equivalent:

(i) The function $\varepsilon$ (or equivalently $\hat{\varepsilon}$ ) defines a causal problem (in the sense of the causality principle);

(ii) There exists $\tilde{\varepsilon}: \mathbb{C} \rightarrow \mathbb{C}$ analytic in the upper half plane $\{z=(x+i y) \in \mathbb{C}, y>0\}$ such that:

$$
\begin{aligned}
& -\exists K>0, \text { such that } \forall y>0, \int_{\mathbb{R}}|\tilde{\varepsilon}(x+i y)|^{2} d x<K \\
& \quad \text { and } \\
& -\hat{\varepsilon}(x)-\varepsilon_{\infty} \text { is the limit of } \tilde{\varepsilon}(z) \text { as } z=x+i y \rightarrow x ;
\end{aligned}
$$

(iii) $\hat{\varepsilon}$ verifies the Kramers-Krönig relations i.e. the real part and imaginary part of $\hat{\varepsilon}$ are conjugate functions, in the sense of Hilbert transforms.

Proof. We directly apply Theorem 2.1 to $\hat{\varepsilon}-\varepsilon_{\infty}$. $\square$

We will use more precisely the following result.

Proposition 2.3. Let $\hat{\varepsilon} \in \varepsilon_{\infty}+L^{2}(\mathbb{R})$ be a complex valued function and $\varepsilon \in$ $\varepsilon_{\infty} \delta+L^{2}(\mathbb{R})$ be defined as the inverse Fourier transform of $\varepsilon$. The two following conditions are equivalent:

(iv) The natural extension of $\hat{\varepsilon}-\varepsilon_{\infty}$ to $\mathbb{C}$ :

$$
\tilde{\varepsilon}: x+i y \mapsto \frac{1}{\sqrt{2 \pi}} \int_{0}^{+\infty} e^{i w(x+i y)}\left(\varepsilon(w)-\varepsilon_{\infty} \delta\right) d w
$$

is analytic in the upper half plane $(y>0)$. Furthermore $\exists K>0, \forall y>0$, $\int_{z \rightarrow x}|\tilde{\varepsilon}(x+i y)|^{2} d x<K$, and $\tilde{\varepsilon}$ converges for a.e. $x \in \mathbb{R}$ towards $\hat{\varepsilon}-\varepsilon_{\infty}$ as 
(v) The problem defined by $\varepsilon$ (or equivalently $\hat{\varepsilon}$ ) is causal.

Proof. This is a consequence of the last theorem.

We can verify easily that the classical dispersive models, such as Drude, DrudeLorentz or Debye are causal. For the special case of the generalized model, this leads us to consider the poles of 2.3. Doing so, it is straightforward to prove that it suffices to impose $b_{l} \geq 0, e_{l} \geq 0$ and $f_{l} \geq 0$. For the rest of the study, we will make this hypothesis.

In the remaining of this paper, we propose to study the generalized model in its full generality. The reader interested in conclusion for classical models may easily find its way.

3. Continuous equations. We consider $\Omega \subset \mathbb{R}^{3}$ an open bounded convex domain, that will be our domain of study. By performing an inverse Fourier transform of (2.3), and after a few basic manipulations, one obtains the following time-domain equations describing the propagation of electromagnetic waves in a generalized dispersive medium $\Omega$ :

$$
\left\{\begin{aligned}
\mu_{0} \frac{\partial \mathbf{H}}{\partial t} & =-\nabla \times \mathbf{E}, & & \\
\varepsilon_{0} \varepsilon_{\infty} \frac{\partial \mathbf{E}}{\partial t} & =\left(\nabla \times \mathbf{H}-\left(\tilde{\mathbf{J}}_{0}+\sum_{l \in L_{1}} \mathbf{J}_{l}+\sum_{l \in L_{2}} \mathcal{J}_{l}\right)\right), & & \\
\tilde{\mathbf{J}}_{0} & =\left(\sigma+\sum_{l \in L_{2}} d_{l}\right) \mathbf{E}, & & \\
\mathbf{J}_{l} & =a_{l} \mathbf{E}-b_{l} \mathbf{P}_{l}, & & \forall l \in L_{1}, \\
\frac{\partial \mathbf{P}_{l}}{\partial t} & =\mathbf{J}_{l}, & & \forall l \in L_{1}, \\
\frac{\partial \mathcal{J}_{l}}{\partial t} & =\left(c_{l}-d_{l} f_{l}\right) \mathbf{E}-f_{l} \mathcal{J}_{l}-e_{l} \mathbf{P}_{l}, & & \forall l \in L_{2}, \\
\frac{\partial \mathbf{P}_{l}}{\partial t} & =d_{l} \mathbf{E}+\mathcal{J}_{l} & & \forall l \in L_{2} .
\end{aligned}\right.
$$

This set of equations will be supplemented with boundary and initial conditions (see next subsection).

REMARK 1. The reader might recognize through this set of equations, Maxwell's equations with several current source terms $\left(\tilde{\boldsymbol{J}}_{0}, \boldsymbol{J}_{l}\right.$ 's, $\mathcal{J}_{l}$ 's) that describe the response of the electrons to the applied electric field. Except for $\tilde{\boldsymbol{J}}_{0}$ that is directly expressed in terms of $\boldsymbol{E}$, the evolution of the polarization currents are driven by ordinary differential equations. This is the standard Auxiliary Differential Equation (ADE) approach as mentioned in the introduction.

These equations will be completed with initial and boundary conditions (see next section).

Let $N$ be the total number of fields involved: $N=2+\operatorname{card}\left(L_{1}\right)+2 \operatorname{card}\left(L_{2}\right)$. Let us write the equation in a more compact way.

We denote by $\boldsymbol{\vartheta}$ the vector of unknowns as $\boldsymbol{\vartheta}=\left(\begin{array}{c}\mathbf{H} \\ \mathbf{E} \\ \left(\mathbf{P}_{l}\right)_{l \in L_{1}} \\ \left(\mathbf{P}_{l}\right)_{l \in L_{2}} \\ \left(\mathcal{J}_{l}\right)_{l \in L_{2}}\end{array}\right)$. 
The differential operator part will be split into two parts $\mathcal{L}$ (containing the Maxwell's differential operator) and $\mathcal{K}$ (as a compact operator perturbation), with:

$$
\mathcal{L}(\boldsymbol{\vartheta})=\left(\begin{array}{c}
-\nabla \times \mathbf{E} \\
\nabla \times \mathbf{H} \\
0 \\
0 \\
0
\end{array}\right), \mathcal{K}(\boldsymbol{\vartheta})=\left(\begin{array}{c}
0 \\
-\left(\sigma+\sum_{l \in L_{2}} d_{l}\right) \mathbf{E}-\sum_{l \in L_{1}}\left(a_{l} \mathbf{E}-b_{l} \mathbf{P}_{l}\right)-\sum_{l \in L_{2}} \mathcal{J}_{l} \\
\left.\left(\frac{1}{a_{l}}\left(a_{l} \mathbf{E}-b_{l} \mathbf{P}_{l}\right)\right)_{l \in L_{1}}\left(\left(c_{l}-d_{l} f_{l}\right) \mathbf{E}-f_{l} \mathcal{J}_{l}-e_{l} \mathbf{P}_{l}\right)\right)_{l \in L_{2}} \\
\left(\frac{e_{l}}{c_{l}+d_{l} f_{l} f_{l}}\left(d_{l} \mathbf{E}+\mathcal{J}_{l}\right)\right)_{l \in L_{2}}
\end{array}\right) .
$$

Thus the equations reformulate as

$$
\Lambda \frac{d \boldsymbol{\vartheta}}{d t}=\mathcal{L}(\boldsymbol{\vartheta})+\mathcal{K}(\boldsymbol{\vartheta})
$$

with

$\Lambda=\operatorname{diag}\left(\mu_{0}, \varepsilon_{\infty} \varepsilon_{0}, \frac{b_{1}}{a_{1}}, \cdots, \frac{b_{L_{1}}}{a_{L_{1}}}, \frac{1}{c_{1}+d_{1} f_{1}}, \cdots, \frac{1}{c_{L_{1}}+d_{L_{1}} f_{L_{1}}}, \frac{e_{1}}{c_{1}+d_{1} f_{1}}, \cdots, \frac{e_{L_{2}}}{c_{L_{2}}+d_{L_{2}} f_{L_{2}}}\right)$.

Let us define $\mathcal{I}=\mathcal{L}+\mathcal{K}$, so that $(3.2)$ becomes

$$
\Lambda \frac{d \boldsymbol{\vartheta}}{d t}=\mathcal{I}(\boldsymbol{\vartheta})
$$

In the rest of the study, we impose that $\left(a_{i}\right)_{i \in\left[0, L_{1}\right]},\left(b_{i}\right)_{i \in\left[0, L_{1}\right]},\left(c_{i}\right)_{i \in\left[0, L_{2}\right]}$ are positive and $\left(d_{i}\right)_{i \in\left[0, L_{2}\right]}$ is non negative.

REMARK 2. If the contribution of one of the pole is zero, one can adapt all the results, same if the positivity assumption is not verified.

3.1. Existence and uniqueness of the solution. We use classical operator theory (see CH90, Paz83, AH03 for details and techniques) to prove existence of a solution to 3.2). Indeed, the latter can be viewed as a compact perturbation of a maximal dissipative operator. Let us first define the different spaces on which our analysis relies. $\mathbf{L}^{2}(\Omega):=L^{2}(\Omega)^{3}$ is the space of square integrable vectorial functions on $\Omega \subset \mathbb{R}^{3}$. $\mathcal{H}(\operatorname{curl}, \Omega)=\left\{\mathbf{V} \in \mathbf{L}^{2}(\Omega) \mid \operatorname{curl}(\mathbf{V}) \in \mathbf{L}^{2}(\Omega\}, \mathcal{H}_{0}(\operatorname{curl}, \Omega)\right.$ the subspace of $\mathcal{H}($ curl,$\Omega)$ with vanishing tangential trace on the boundary of $\Omega$. $\mathcal{D}(\mathcal{L})=\mathcal{D}(\mathcal{I})=$ $\mathcal{H}(\operatorname{curl}, \Omega) \times \mathcal{H}_{0}(\operatorname{curl}, \Omega) \times \mathbf{L}^{2}(\Omega)^{N-2}, \mathcal{D}(\mathcal{K})=\left(\mathbf{L}^{2}(\Omega)\right)^{N}$ the domain of $\mathcal{L}, \mathcal{I}, \mathcal{K}$ respectively. One writes the equations (in a strong formulation) in a system form:

$$
\left\{\begin{array}{l}
\Lambda \frac{d \boldsymbol{\vartheta}}{d t}=\mathcal{L}(\boldsymbol{\vartheta})+\mathcal{K}(\boldsymbol{\vartheta}), \\
\boldsymbol{\vartheta}(0)=U^{0}
\end{array}\right.
$$

In this framework, let us define the classical $\mathbf{L}^{2}(\Omega)$ real scalar product, denoted by $\langle\cdot, \cdot \cdot\rangle$ and the associated norm $\|\cdot\|$. We also define the following weighted scalar product on $\mathcal{D}(\mathcal{K})=\left(\mathbf{L}^{2}(\Omega)\right)^{N}$

$$
\langle\boldsymbol{\vartheta}, \boldsymbol{\varsigma}\rangle_{\Lambda}=\langle\Lambda \boldsymbol{\vartheta}, \boldsymbol{\varsigma}\rangle, \forall(\boldsymbol{\vartheta}, \boldsymbol{\varsigma}) \in D(\mathcal{K}),
$$


with the associated norm

$$
\|\boldsymbol{\vartheta}\|_{\Lambda}=\langle\boldsymbol{\vartheta}, \boldsymbol{\vartheta}\rangle_{\Lambda}^{\frac{1}{2}}
$$

For $T>0$, we are interested in the existence and uniqueness to the Cauchy problem (3.4) in $[0, T]$.

LEMMA 3.1.

(i) The unbounded operator $\mathcal{L}$ is defined on $D(\mathcal{L})=\mathcal{H}($ curl,$\Omega) \times \mathcal{H}_{0}($ curl,$\Omega) \times$ $\boldsymbol{L}^{2}(\Omega)^{N-2}$ which is dense in $\boldsymbol{L}^{2}(\Omega)^{N}$. Furthermore, $\mathcal{L}$ is dissipative in $\boldsymbol{L}^{2}(\Omega)^{N}$ and

$$
\langle\mathcal{L}(\boldsymbol{\vartheta}), \boldsymbol{\vartheta}\rangle=0, \quad \forall \boldsymbol{\vartheta} \in D(\mathcal{L})
$$

(ii) The operator $\mathcal{K}$ is defined on $D(\mathcal{K})=\boldsymbol{L}^{2}(\Omega)^{N}$. Furthermore,

$$
\text { with } C_{L_{2}}=\max _{l \in L_{2}} d_{l} \sqrt{\frac{\left.e_{l}(\boldsymbol{\vartheta}), \boldsymbol{\vartheta}\right\rangle}{\varepsilon_{\infty}\left(c_{l}+d_{l} f_{l}\right)}} . C_{L_{2}}\|\boldsymbol{\vartheta}\|_{\Lambda}^{2},
$$

Proof. For (i): From the Green formula for the curl operator, one has

$$
\langle\mathcal{L}(\boldsymbol{\vartheta}), \boldsymbol{\varsigma}\rangle=-\langle\boldsymbol{\vartheta}, \mathcal{L}(\boldsymbol{\varsigma})\rangle \quad \forall(\boldsymbol{\vartheta}, \boldsymbol{\varsigma}) \in D(\mathcal{L})^{2} .
$$

This yields that

$$
\mathcal{L}^{*}=-\mathcal{L}, \quad D\left(\mathcal{L}^{*}\right)=D(\mathcal{L})
$$

and thus

$$
\langle\mathcal{L}(\boldsymbol{\vartheta}), \boldsymbol{\vartheta}\rangle=0, \quad \forall \boldsymbol{\vartheta} \in D(\mathcal{L}) .
$$

This implies that $\mathcal{L}$ is dissipative in $\mathbf{L}^{2}(\Omega)^{N}$.

For (ii): One has

$$
\begin{aligned}
\langle\mathcal{K}(\boldsymbol{\vartheta}), \boldsymbol{\vartheta}\rangle & =-\left(\sigma+\sum_{l \in L_{2}} \frac{c_{l} d_{l}}{c_{l}+d_{l} f_{l}}\right)\|\mathbf{E}\|_{L^{2}(\Omega)}^{2} \\
& -\sum_{l \in L_{2}} \frac{f_{l}}{c_{l}+d_{l} f_{l}}\left\|\mathcal{J}_{l}+d_{l} \mathbf{E}\right\|_{L^{2}(\Omega)}^{2}+\sum_{l \in L_{2}} \frac{e_{l} d_{l}}{c_{l}+d_{l} f_{l}}\left\langle\mathbf{E}, \mathbf{P}_{l}\right\rangle \\
& -\sum_{l \in L_{1}} \frac{1}{a_{l}}\left\|a_{l} \mathbf{E}-b_{l} \mathbf{P}_{l}\right\|^{2} .
\end{aligned}
$$

Thus

$$
\begin{aligned}
\langle\mathcal{K}(\boldsymbol{\vartheta}), \boldsymbol{\vartheta}\rangle & \leq \sum_{l \in L_{2}} \frac{e_{l} d_{l}}{c_{l}+d_{l} f_{l}}\left\langle\mathbf{E}, \mathbf{P}_{l}\right\rangle, \\
\langle\mathcal{K}(\boldsymbol{\vartheta}), \boldsymbol{\vartheta}\rangle & \leq\left(\max _{l \in L_{2}} d_{l} \sqrt{\frac{e_{l}}{\varepsilon_{\infty}\left(c_{l}+d_{l} f_{l}\right)}}\right)\|\boldsymbol{\vartheta}\|_{\Lambda}^{2} .
\end{aligned}
$$

REMARK 3. One might notice that $d_{l}=0$ or $e_{l}=0$ implies that

$$
\langle\mathcal{K}(\boldsymbol{\vartheta}), \boldsymbol{\vartheta}\rangle \leq 0 .
$$


The rest of the proof of existence goes along the same lines as in [LS13] and consists in a straightforward generalization of the result obtained in the latter reference. We will thus not detail the proof here, but simply state the final result.

Theorem 3.2. If $\boldsymbol{\vartheta}_{0} \in\left(\boldsymbol{H}^{0}, \boldsymbol{E}^{0},\left(\boldsymbol{P}_{l}^{0}\right)_{l},\left(\mathcal{J}_{l}^{0}\right)_{l}\right) \in D(\mathcal{I})$, then there exists a unique weak solution:

$$
\boldsymbol{\vartheta}=\left(\boldsymbol{H}, \boldsymbol{E},\left(\boldsymbol{P}_{l}\right)_{l},\left(\mathcal{J}_{l}\right)_{l}\right) \in \mathcal{C}^{0}([0, T], D(\mathcal{I})) \cap \mathcal{C}^{1}\left([0, T], \boldsymbol{L}^{2}(\Omega)^{N}\right),
$$

of equation (3.4).

3.2. Energy. We define the energy associated to the system (3.4) at a given time $t \in[0, T]$ as follows:

$$
\begin{aligned}
\xi(t) & =\frac{1}{2}\left(\|\mathbf{H}(t)\|_{L^{2}(\Omega)}^{2}+\varepsilon_{\infty}\|\mathbf{E}(t)\|_{L^{2}(\Omega)}^{2}+\sum_{l \in L_{1}} \frac{b_{l}}{a_{l}}\left\|\mathbf{P}_{l}(t)\right\|_{L^{2}(\Omega)}^{2}\right. \\
& \left.+\sum_{l \in L_{2}} \frac{e_{l}}{c_{l}+d_{l} f_{l}}\left\|\mathbf{P}_{l}(t)\right\|_{L^{2}(\Omega)}^{2}+\sum_{l \in L_{2}} \frac{1}{c_{l}+d_{l} f_{l}}\left\|\mathcal{J}_{l}(t)\right\|_{L^{2}(\Omega)}^{2}\right) .
\end{aligned}
$$

In other words,

$$
\xi(t)=\frac{1}{2}\|\boldsymbol{\vartheta}\|_{\Lambda}^{2} .
$$

The following results states the boundedness of the energy.

TheOREM 3.3. If $\boldsymbol{\vartheta} \in \mathcal{C}^{0}([0, T], D(\mathcal{I})) \cap \mathcal{C}^{1}\left(0, T,\left(\boldsymbol{L}^{2}(\Omega)\right)^{N}\right), \xi$ is bounded on $[0, T]$.

Proof. Assuming a sufficient regularity for each of the fields, using equation 3.4 leads to:

$$
\left\langle\Lambda \frac{\partial \boldsymbol{\vartheta}}{\partial t}, \boldsymbol{\vartheta}\right\rangle=\langle\mathcal{L}(\boldsymbol{\vartheta}), \boldsymbol{\vartheta}\rangle+\langle\mathcal{K}(\boldsymbol{\vartheta}), \boldsymbol{\vartheta}\rangle,
$$

so that by lemma 3.1, $\forall t \in[0, T]$ :

$$
\frac{d \xi}{d t}(t)=\langle\mathcal{K}(\boldsymbol{\vartheta}(t)), \boldsymbol{\vartheta}(t)\rangle \leq 2 C_{L_{2}} \xi(t)
$$

which directly implies that, $\forall t \in[0, T], \xi(t) \leqslant \xi(0) \exp \left(2 C_{L_{2}} t\right)$.

\section{Discontinuous Galerkin discretization.}

4.1. Formulation and preliminary results. We discretize the system using a discontinuous Galerkin method formulated on simplicial meshes. We follow the approach of [FLLP05]. For the analysis, we suppose that the domain $\Omega$ is furthermore polyhedral. Let $\Omega_{h}$ be a discretization of the computational domain $\Omega$, using a tessellation $\mathcal{T}_{h}=\left(\tau_{i}\right)_{i \in\left[0, N_{\mathcal{T}_{h}}\right]}, N_{\mathcal{T}_{h}} \in \mathbb{N}^{*}$, such that $\Omega_{h} \equiv \Omega$. For $i \in\left[0, N_{\mathcal{T}_{h}}\right]$, we define $h_{i}$ the diameter of the tetrahedron $\tau_{i}$ and $h=\max _{i \in\left[0, N_{\mathcal{T}_{h}}\right]} h_{i}$. An internal face is denoted by $a_{i k}=\tau_{i} \cap \tau_{k}$, for $i \neq k$ and $\mathbf{n}_{i k}$ is the unitary normal vector oriented from $\tau_{i}$ to $\tau_{k}$. For a given element $\tau_{i}, i \in\left[0, N_{\mathcal{T}_{h}}\right]$, one denotes by $\mathcal{V}_{i}$ the set of the indices of all neighboring elements of $\tau_{i} . \mathcal{F}_{h}^{\text {int }}$ will denote the set of all internal faces of the mesh. We assume that the mesh is quasi uniform and one assumes the inverse assumption, i.e. there exists a positive constant $\eta$ (independent of $h$ ) such that $\forall h, \forall \tau_{i} \in \mathcal{T}_{h}, \forall k \in \mathcal{V}_{i}, \frac{h_{i}}{h_{k}} \leq \eta$. 
General setting. One seeks an approximation $\boldsymbol{\vartheta}_{h}$ of $\boldsymbol{\vartheta}$ such that on each $\tau_{i}, i \in$ $\left[0, N_{\mathcal{T}_{h}}\right]$, the components of $\boldsymbol{\vartheta}_{h}$ are polynomials whose degree can in all generality depend on the triangle $\tau_{i}$. For $i$ given in $\left[0, N_{\mathcal{T}_{h}}\right]$, we denote by $d_{i}$ the number of degrees of freedom associated to the finite element $\tau_{i}$ and by $\mathcal{P}_{i}$ the associated polynomial space. Moreover, $\left(\varphi_{i, j}\right)_{1 \leq j \leq d_{i}}$ defines a set of linearly independent functions such that $\mathcal{P}_{i}=\operatorname{Span}\left\{\varphi_{i, j}, 1 \leq j \leq d_{i}\right\}$. We thus define:

$$
\mathbf{V}_{h}=\left\{\mathbf{W}_{h} \in \mathbf{L}^{2}(\Omega) \quad \mid \quad\left(\mathbf{W}_{h}\right)_{/ \tau_{i}} \in\left(\mathcal{P}_{i}\right)^{3}, \quad \forall \tau_{i} \in \mathcal{T}_{h}\right\}
$$

the corresponding approximation space.

For any $\mathbf{W}_{h} \in \mathbf{V}_{h}$ :

- $\mathbf{W}_{i}$ denotes its restriction to the element $\tau_{i}$.

- We define its average through any internal face $a_{i k}$, for given $(i, k)$ as:

$$
\left\{\mathbf{W}_{h}\right\}_{i k}=\frac{\mathbf{W}_{i / a_{i k}}+\mathbf{W}_{k / a_{i k}}}{2},
$$

where $\mathbf{W}_{i / a_{i k}}$ denotes the restriction of $\mathbf{W}_{i}$ to the face $a_{i k}$.

- $\llbracket \mathbf{W}_{h} \rrbracket_{i k}$ stands for the tangential jump through any internal face $a_{i k}$ :

$$
\llbracket \mathbf{W}_{h} \rrbracket_{i k}=\left(\mathbf{W}_{k / a_{i k}}-\mathbf{W}_{i / a_{i k}}\right) \times \mathbf{n}_{i k} .
$$

We are thus looking for $\boldsymbol{\vartheta}_{h} \in \mathbb{V}_{h}$, where:

$$
\mathbb{V}_{h}=\mathbf{V}_{h}^{N}
$$

In what follows, we consider the particular case where the discretization space is given by piecewise polynomials of degree at most $k$ in each tetrahedron. In $\tau_{i}$, it is denoted by $\mathbb{P}_{k}\left(\tau_{i}\right)$. Then:

$$
\mathbf{V}_{h}=\left\{\mathbf{W}_{h} \in \mathbf{L}^{2}(\Omega) \quad \mid \quad\left(\mathbf{W}_{h}\right)_{/ \tau_{i}} \in\left(\mathbb{P}_{k}\left(\tau_{i}\right)\right)^{3}, \quad \forall \tau_{i} \in \mathcal{T}_{h}\right\} .
$$

For what concerns the treatment of the boundary condition on a metallic wall, for each of the corresponding boundary face (still denoted $a_{i k}$ ), we set $\mathbf{E}_{k / a_{i k}}=-\mathbf{E}_{i / a_{i k}}$, and $\mathbf{H}_{k / a_{i k}}=\mathbf{H}_{i / a_{i k}}$.

Local weak formulation. Let us define, $\forall i \in\left[0, N_{\mathcal{T}_{h}}\right],\langle\cdot, \cdot\rangle_{\tau_{i}}$, the $\mathbf{L}^{2}$-scalar product on $\tau_{i},\langle\cdot, \cdot\rangle_{h}:=\sum_{i \in\left[0, N_{\mathcal{T}_{h}}\right]}\langle\cdot, \cdot\rangle_{\tau_{i}}$, and $\langle\cdot, \cdot\rangle_{\mathcal{F}_{h}^{i n t}}:=\sum_{F \in \mathcal{F}_{h}^{i n t}}\langle\cdot, \cdot\rangle_{F}$, with $\langle\cdot, \cdot\rangle_{F}$ the $L^{2}$ scalar product on the face $F$. Furthermore, we define $\langle\cdot, \cdot\rangle_{\partial \tau_{i}}$ as the $\mathbf{L}^{2}$-scalar product on $\partial \tau_{i}$ that has to be understand as $\sum_{k \in \mathcal{V}_{i}}\langle\cdot, \cdot\rangle_{a_{i k}}$.

For all $\boldsymbol{\vartheta} \in \mathbf{L}^{2}(\Omega)^{N}$, we write $\boldsymbol{\vartheta}=\left(\begin{array}{c}\boldsymbol{\vartheta}_{1} \\ \boldsymbol{\vartheta}_{2} \\ \hat{\boldsymbol{\vartheta}}\end{array}\right)$, with $\left(\boldsymbol{\vartheta}_{1}, \boldsymbol{\vartheta}_{2}\right) \in \mathbf{L}^{2}(\Omega) \times \mathbf{L}^{2}(\Omega)$, $\hat{\boldsymbol{\vartheta}} \in \mathbf{L}^{2}(\Omega)^{N-2}$. Finally, for each $i \in\left[0, N_{\mathcal{T}_{h}}\right], \hat{\mathbf{n}}_{i} \in \mathbb{R}^{3}$ is defined on $\partial \tau_{i}$ and $\forall k \in \mathcal{V}_{i}$, $\hat{\mathbf{n}}_{i}=\mathbf{n}_{i k}$ on $a_{i k}$.

The formulation of the discrete problem follows a discontinuous Galerkin approach in the spirit of FLLP05, LS13, LSV13: we locally (i.e. on each tetrahedron) perform an integration by part and define a notion of fluxes to recover the communication at the interfaces of the mesh. In the latter references, the focus was made on centered 
fluxes. In this work, we allow for more general fluxes (from centered to upwind), in this purpose, we fix $\alpha \in[0,1]$. The local formulation thus writes as follows: Find $\boldsymbol{\vartheta}_{h} \in \mathbb{V}_{h}$ such that $\forall i \in\left[0, N_{\mathcal{T}_{h}}\right], \forall \boldsymbol{\vartheta}^{\prime}{ }_{h} \in \mathbb{V}_{h}$,

$$
\left\langle\Lambda \frac{\partial}{\partial t} \boldsymbol{\vartheta}_{h}, \boldsymbol{\vartheta}_{h}^{\prime}\right\rangle_{\tau_{i}}=\left\langle\boldsymbol{\vartheta}_{h}, \mathcal{L}_{i}^{*}\left(\boldsymbol{\vartheta}_{h}^{\prime}\right)\right\rangle_{\tau_{i}}-\left\langle\mathbf{F}_{i, h}^{\alpha}\left(\boldsymbol{\vartheta}_{h}\right) \cdot \hat{\mathbf{n}}_{i}, \boldsymbol{\vartheta}_{h}^{\prime}\right\rangle_{\partial \tau_{i}}+\left\langle\mathcal{K}\left(\boldsymbol{\vartheta}_{h}\right), \boldsymbol{\vartheta}_{h}^{\prime}\right\rangle_{\tau_{i}} \text {, on }[0, T],
$$

where $\forall i \in\left[0, N_{\mathcal{T}_{h}}\right], \forall \vartheta \in \mathbb{V}_{h}$ :

$$
\mathcal{L}_{i}^{*}(\boldsymbol{\vartheta})=\left(\begin{array}{c}
\nabla \times\left(\boldsymbol{\vartheta}_{2 \mid \tau_{i}}\right) \\
-\nabla \times\left(\boldsymbol{\vartheta}_{1 \mid \tau_{i}}\right) \\
\mathbf{0}
\end{array}\right)
$$

and the numerical flux $\mathbf{F}_{i, h}^{\alpha}$ is defined on $\partial \tau_{i}$, by:

$$
\mathbf{F}_{i, h}^{\alpha}(\boldsymbol{\vartheta})_{\mid \partial \tau_{i}}=\left(\begin{array}{c}
\frac{1}{\bar{Y}}\left(\hat{\mathbf{n}}_{i} \times\left\{Y \boldsymbol{\vartheta}_{2}\right\}+\frac{1}{2} \alpha\left(\hat{\mathbf{n}}_{i} \times \hat{\mathbf{n}}_{i} \times \llbracket \boldsymbol{\vartheta}_{1} \rrbracket\right)\right) \\
\frac{1}{\bar{Z}}\left(-\hat{\mathbf{n}}_{i} \times\left\{Z \boldsymbol{\vartheta}_{1}\right\}+\frac{1}{2} \alpha\left(\hat{\mathbf{n}}_{i} \times \hat{\mathbf{n}}_{i} \times \llbracket \boldsymbol{\vartheta}_{2} \rrbracket\right)\right) .
\end{array}\right) .
$$

Here $\{\cdot\}$ and $\llbracket \cdot \rrbracket$ have to be understand as the corresponding part of (respectively) the average and the jump on the corresponding faces of $\partial \tau_{i}$.

The above definition allows for a discontinuous set of coefficients across the interface, by including the possible jump of the impedance and admittance and their mean across the interface $(\bar{Y}, \bar{Z})$. In the theoretical setting we propose, we choose, as a first approach, to consider only the case of a homogeneous dispersive medium with constant coefficients (i.e. $Z=\sqrt{\frac{\mu_{0}}{\varepsilon_{0} \varepsilon_{\infty}}}$ and $Y=\frac{1}{Z}$. ). Thus this degenerates in a easier expression, but we wanted to keep it general so that the reader may easily find its way through the generalization of some of the results proved here.

REMARK 4. If $\alpha=0$, the flux corresponds to the classical centered flux. If $\alpha=1$, the flux is the classical upwind flux.

Global weak formulation. We define $\forall \boldsymbol{\vartheta} \in \mathbb{V}_{h}$ :

$$
\mathcal{L}_{h}(\boldsymbol{\vartheta})=-\mathcal{L}_{h}^{*}(\boldsymbol{\vartheta})=\left(\begin{array}{c}
-\nabla_{h} \times \boldsymbol{\vartheta}_{2} \\
\nabla_{h} \times \boldsymbol{\vartheta}_{1} \\
\mathbf{0}
\end{array}\right),
$$

with $\nabla_{h}$ the piecewise curl operator defined on each $\tau_{i}$ for $i \in\left[0, N_{\mathcal{T}_{h}}\right]$ and for all $\mathbf{b}_{h} \in \mathbf{V}_{h}$ as:

$$
\left(\nabla_{h} \times \mathbf{b}_{h}\right)_{/ \tau_{i}}=\nabla \times\left(\mathbf{b}_{h / \tau_{i}}\right) .
$$

Furthermore, we will need the bilinear forms $m, a, b_{\alpha}, k$ defined on $\mathbb{V}_{h} \times \mathbb{V}_{h}$ as follows $\forall\left(\boldsymbol{\vartheta}, \boldsymbol{\vartheta}^{\prime}\right) \in \mathbb{V}_{h} \times \mathbb{V}_{h}$ :

$$
\left\{\begin{array}{l}
m\left(\boldsymbol{\vartheta}, \boldsymbol{\vartheta}^{\prime}\right)=\left\langle\boldsymbol{\vartheta}, \boldsymbol{\vartheta}^{\prime}\right\rangle_{\Lambda}, \\
a\left(\boldsymbol{\vartheta}, \boldsymbol{\vartheta}^{\prime}\right)=\left\langle\boldsymbol{\vartheta}, \mathcal{L}_{h}^{*}\left(\boldsymbol{\vartheta}^{\prime}\right)\right\rangle_{h}, \\
b_{\alpha}\left(\boldsymbol{\vartheta}, \boldsymbol{\vartheta}^{\prime}\right)=-\frac{1}{\bar{Z}}\left[\left\langle\left\{Z \boldsymbol{\vartheta}_{1}\right\}, \llbracket \boldsymbol{\vartheta}_{2}^{\prime} \rrbracket\right\rangle_{\mathcal{F}_{h}^{\text {int }}}+\alpha\left\langle\llbracket \boldsymbol{\vartheta}_{2} \rrbracket, \llbracket \boldsymbol{\vartheta}_{2}^{\prime} \rrbracket\right\rangle_{\mathcal{F}_{h}^{\text {int }}}\right] \\
+\frac{1}{\bar{Y}}\left[\left\langle\left\{Y \boldsymbol{\vartheta}_{2}\right\} \llbracket, \boldsymbol{\vartheta}_{1}^{\prime} \rrbracket\right\rangle_{\mathcal{F}_{h}^{\text {int }}}-\alpha\left\langle\llbracket \llbracket \boldsymbol{\vartheta}_{1} \rrbracket \llbracket \boldsymbol{\vartheta}_{1}^{\prime} \rrbracket\right\rangle_{\mathcal{F}_{h}^{\text {int }}}\right]+\int_{\partial \Omega} \boldsymbol{\vartheta}_{2}^{\prime} \cdot\left(\boldsymbol{\vartheta}_{1} \times \mathbf{n}\right) \\
k\left(\boldsymbol{\vartheta}, \boldsymbol{\vartheta}^{\prime}\right)=\left\langle\mathcal{K}(\boldsymbol{\vartheta}), \boldsymbol{\vartheta}^{\prime}\right\rangle_{h} .
\end{array}\right.
$$


We can then recast the global semi-discrete weak formulation as:

Find $\boldsymbol{\vartheta}_{h} \in \mathbb{V}_{h}$ such that

$$
m\left(\frac{\partial \boldsymbol{\vartheta}_{h}}{\partial t}, \boldsymbol{\vartheta}_{h}^{\prime}\right)=a\left(\boldsymbol{\vartheta}_{h}, \boldsymbol{\vartheta}_{h}^{\prime}\right)+b_{\alpha}\left(\boldsymbol{\vartheta}_{h}, \boldsymbol{\vartheta}_{h}^{\prime}\right)+k\left(\boldsymbol{\vartheta}_{h}, \boldsymbol{\vartheta}_{h}^{\prime}\right), \forall \boldsymbol{\vartheta}_{h}^{\prime} \in \mathbb{V}_{h}
$$

We establish a consistency result.

Proposition 4.1 (Consistency). The exact solution $\vartheta$ of 3.2 verifies:

$$
m\left(\frac{\partial \boldsymbol{\vartheta}}{\partial t}, \boldsymbol{\vartheta}_{h}^{\prime}\right)=a\left(\boldsymbol{\vartheta}, \boldsymbol{\vartheta}_{h}^{\prime}\right)+b_{\alpha}\left(\boldsymbol{\vartheta}, \boldsymbol{\vartheta}_{h}^{\prime}\right)+k\left(\boldsymbol{\vartheta}, \boldsymbol{\vartheta}_{h}^{\prime}\right), \forall \boldsymbol{\vartheta}_{h}^{\prime} \in \mathbb{V}_{h}
$$

Proof. The proof presents no difficulties. The main question is to justify that $a\left(\boldsymbol{\vartheta}, \boldsymbol{\vartheta}_{h}^{\prime}\right)+b\left(\boldsymbol{\vartheta}, \boldsymbol{\vartheta}_{h}^{\prime}\right)=\left\langle\mathcal{L}_{h}(\boldsymbol{\vartheta}), \boldsymbol{\vartheta}_{h}^{\prime}\right\rangle$. This is the case since $\boldsymbol{\vartheta}$ has a continuous tangential trace across the interface. The result follows.

Lemma 4.2. For $\alpha \in[0,1]$, then $\forall \boldsymbol{\vartheta}_{h} \in \mathbb{V}_{h}$ :

$$
a\left(\boldsymbol{\vartheta}_{h}, \boldsymbol{\vartheta}_{h}\right)+b_{\alpha}\left(\boldsymbol{\vartheta}_{h}, \boldsymbol{\vartheta}_{h}\right)=-\alpha\left|\boldsymbol{\vartheta}_{h}\right|_{S}^{2} \leq 0,
$$

where we define

$$
\left|\boldsymbol{\vartheta}_{h}\right|_{S}^{2}:=\left(\frac{1}{\bar{Y}}\left\|\llbracket \llbracket \boldsymbol{\vartheta}_{1} \rrbracket\right\|_{\mathcal{F}_{h}^{\text {int }}}^{2}+\frac{1}{\bar{Z}}\left\|\llbracket \boldsymbol{\vartheta}_{2} \rrbracket\right\|_{\mathcal{F}_{h}^{\text {int }}}^{2}\right)
$$

Proof. If $F$ is in $\mathcal{F}_{h}^{\text {int }}$, then there exist $(i, k) \in\left[0, N_{\mathcal{T}_{h}}\right] \times\left[0, N_{\mathcal{T}_{h}}\right]$ such that $F=a_{i k}$. In this proof, let $\mathbf{n}$ denotes the normal to $F$ oriented from $\tau_{i}$ to $\tau_{k}$ (w.l.o.g), and $\boldsymbol{\vartheta}^{+}$ the value $\boldsymbol{\vartheta}_{i / a_{i k}}, \boldsymbol{\vartheta}^{-}$the value $\boldsymbol{\vartheta}_{k / a_{i k}}$, with analogous definitions for $Y^{+}$and $Y^{-}, Z^{+}$ and $Z^{-}$. We have that:

$$
\begin{aligned}
a\left(\boldsymbol{\vartheta}_{h}, \boldsymbol{\vartheta}_{h}\right)+b_{\alpha}\left(\boldsymbol{\vartheta}_{h}, \boldsymbol{\vartheta}_{h}\right)= & \left\langle\boldsymbol{\vartheta}_{1}^{+} \times \mathbf{n}, \boldsymbol{\vartheta}_{2}^{+}\right\rangle_{\mathcal{F}_{h}^{\text {int }}}-\left\langle\boldsymbol{\vartheta}_{1}^{-} \times \mathbf{n}, \boldsymbol{\vartheta}_{2}^{-}\right\rangle_{\mathcal{F}_{h}^{\text {int }}}+b_{\alpha}\left(\boldsymbol{\vartheta}_{h}, \boldsymbol{\vartheta}_{h}\right) \\
= & -\frac{1}{2 \bar{Z}}\left[\left\langle Z^{+} \boldsymbol{\vartheta}_{1}^{+}, \boldsymbol{\vartheta}_{2}^{+} \times \mathbf{n}\right\rangle_{\mathcal{F}_{h}^{\text {int }}}+\left\langle Z^{-} \boldsymbol{\vartheta}_{1}^{-}, \boldsymbol{\vartheta}_{2}^{+} \times \mathbf{n}\right\rangle_{\mathcal{F}_{h}^{\text {int }}}\right. \\
& \left.-\left\langle Z^{+} \boldsymbol{\vartheta}_{1}^{+}, \boldsymbol{\vartheta}_{2}^{-} \times \mathbf{n}\right\rangle_{\mathcal{F}_{h}^{\text {int }}}-\left\langle Z^{-} \boldsymbol{\vartheta}_{1}^{-}, \boldsymbol{\vartheta}_{2}^{-} \times \mathbf{n}\right\rangle_{\mathcal{F}_{h}^{\text {int }}}\right] \\
& +\frac{1}{2 \bar{Y}}\left[\left\langle Y^{+} \boldsymbol{\vartheta}_{2}^{+}, \boldsymbol{\vartheta}_{1}^{+} \times \mathbf{n}\right\rangle_{\mathcal{F}_{h}^{i n t}}+\left\langle Y^{-} \boldsymbol{\vartheta}_{2}^{-}, \boldsymbol{\vartheta}_{1}^{+} \times \mathbf{n}\right\rangle_{\mathcal{F}_{h}^{\text {int }}}\right. \\
& \left.-\left\langle Y^{+} \boldsymbol{\vartheta}_{2}^{+}, \boldsymbol{\vartheta}_{1}^{-} \times \mathbf{n}\right\rangle_{\mathcal{F}_{h}^{\text {int }}}-\left\langle Y^{-} \boldsymbol{\vartheta}_{2}^{-}, \boldsymbol{\vartheta}_{1}^{-} \times \mathbf{n}\right\rangle_{\mathcal{F}_{h}^{\text {int }}}\right] \\
& +\left\langle\boldsymbol{\vartheta}_{1}^{+} \times \mathbf{n}, \boldsymbol{\vartheta}_{2}^{+}\right\rangle_{\mathcal{F}_{h}^{\text {int }}}-\left\langle\boldsymbol{\vartheta}_{1}^{-} \times \mathbf{n}, \boldsymbol{\vartheta}_{2}^{-}\right\rangle_{\mathcal{F}_{h}^{\text {int }}} \\
& -\alpha\left(\frac{1}{\bar{Y}}\left\|\llbracket \boldsymbol{\vartheta}_{1} \rrbracket\right\|_{\mathcal{F}_{h}^{\text {int }}}^{2}+\frac{1}{\bar{Z}}\left\|\llbracket \boldsymbol{\vartheta}_{2} \rrbracket\right\|_{\mathcal{F}_{h}^{\text {int }}}^{2}\right) .
\end{aligned}
$$

Using the basic relations between $\bar{Y}, \bar{Z}, Z^{ \pm}, Y^{ \pm}$and naturally grouping terms, we find that all the terms except the ones in factor of $\alpha$ vanishes. Thus,

$$
a\left(\boldsymbol{\vartheta}_{h}, \boldsymbol{\vartheta}_{h}\right)+b_{\alpha}\left(\boldsymbol{\vartheta}_{h}, \boldsymbol{\vartheta}_{h}\right)=-\alpha\left(\frac{1}{\bar{Y}}\left\|\llbracket \boldsymbol{\vartheta}_{1} \rrbracket\right\|_{\mathcal{F}_{h}^{\text {int }}}^{2}+\frac{1}{\bar{Z}}\left\|\llbracket \boldsymbol{\vartheta}_{2} \rrbracket\right\|_{\mathcal{F}_{h}^{\text {int }}}^{2}\right) .
$$

The result follows.

We complete the set of useful lemma by the so called inverse inequalities. 
Lemma 4.3. Let $\alpha \in[0,1]$. One has for all $\varsigma \in \mathbb{V}_{h}$ :

$$
\sup _{\boldsymbol{\xi} \in \mathbb{V}_{h}} \frac{\left|a(\boldsymbol{\varsigma}, \boldsymbol{\xi})+b_{\alpha}(\boldsymbol{\varsigma}, \boldsymbol{\xi})\right|}{\|\boldsymbol{\xi}\|} \leq C h^{-1} \eta\|\boldsymbol{\varsigma}\|
$$

and

$$
\sup _{\boldsymbol{\xi} \in \mathbb{V}_{h}} \frac{|k(\boldsymbol{\varsigma}, \boldsymbol{\xi})|}{\|\boldsymbol{\xi}\|} \leq C\|\boldsymbol{\varsigma}\|
$$

Similarly

$$
\sup _{\boldsymbol{\xi} \in \mathbb{V}_{h}} \frac{\left|b_{\alpha}(\boldsymbol{\varsigma}, \boldsymbol{\xi})\right|}{\|\boldsymbol{\xi}\|} \leq C \eta h^{-1}\|\boldsymbol{\varsigma}\|
$$

and finally $\forall(\varsigma, \xi) \in \mathbb{V}_{h} \times \mathbb{V}_{h}$,

$$
\left|b_{\alpha}(\boldsymbol{\varsigma}, \boldsymbol{\xi})\right| \leq C \eta h^{-\frac{1}{2}}\|\boldsymbol{\varsigma}\||\boldsymbol{\xi}|_{S},
$$

where $\eta$ is the regularity mesh parameter (linked to quasi-uniformity).

Proof. The proof is classical. The first (and third, fourth) inequality relies on inverse estimate on each $\tau_{i}$ (and $\partial \tau_{i}$ ) and on the assumption that the mesh is quasiuniform. The second inequality only expresses that the operator $\mathcal{K}$ is bounded.

Energy principle. We are interested in writing a semi-discrete energy principle for this set of equations.

Proposition 4.4. If $\boldsymbol{\vartheta}_{h} \in \mathbb{V}_{h}$ is solution of the semi discrete scheme, the semidiscrete energy $\mathcal{E}_{h}$ defined on $[0, T]$ by:

$$
\mathcal{E}_{h}=\frac{1}{2} m\left(\boldsymbol{\vartheta}_{h}, \boldsymbol{\vartheta}_{h}\right)=\frac{1}{2}\left\|\boldsymbol{\vartheta}_{h}\right\|_{\Lambda}^{2},
$$

is bounded in finite time.

Proof. One has using Lemma 4.2 that there exists $C>0$ such that on $[0, T]$ :

$$
m\left(\frac{\partial \boldsymbol{\vartheta}_{h}}{\partial t}, \boldsymbol{\vartheta}_{h}\right) \leq k\left(\boldsymbol{\vartheta}_{h}, \boldsymbol{\vartheta}_{h}\right) \leq C\left\|\boldsymbol{\vartheta}_{h}\right\|_{\Lambda}^{2}
$$

This gives $\frac{\partial \mathcal{E}_{h}}{\partial t} \leq C \mathcal{E}_{h}$ that implies $\mathcal{E}_{h}(t) \leq \exp (C t) \mathcal{E}_{h}(0), \forall t \in[0, T]$.

4.2. Convergence of the semi-discrete scheme. To prove the convergence, we will furthermore suppose that initial conditions as stated in (3.4) are regular enough so that there exists $s>1$ such that $\boldsymbol{\vartheta} \in \mathcal{C}^{0}\left(0, T, \mathbf{H}^{s}(\Omega)^{N}\right)$. The structure of the semidiscrete scheme is the same as in [LS13] up to the difference that the analogous expression of $\langle\mathcal{K} \boldsymbol{\vartheta}, \boldsymbol{\vartheta}\rangle$ is negative in [LS13] and that here we allow for a more general definition of fluxes. Despite these notable differences, one can follow the key ideas of the latter reference to prove the semi-convergence of the scheme. First, we define $\pi_{h}$, the $\mathbf{L}^{2}$ projection on $\mathbb{V}_{h}$. We recall that for all $\boldsymbol{v} \in\left(\mathbf{H}^{s}(\Omega)\right)^{N}$ :

$$
\left\|\boldsymbol{v}-\pi_{h}(\boldsymbol{v})\right\| \leq C h^{\min (s, k)+1}|\boldsymbol{v}|_{\mathbf{H}^{s}(\Omega)^{N}} .
$$

In this section, we denote $\alpha \lesssim \beta$, to express that there exists $C>0$ (independent of the mesh size $h$ and time $t \in[0, T])$ such that $\alpha \leq C \beta$. We have on $[0, T]$ : 


$$
\begin{aligned}
m\left(\partial_{t}\left(\boldsymbol{\vartheta}_{h}-\pi_{h}(\boldsymbol{\vartheta})\right), \boldsymbol{\vartheta}_{h}^{\prime}\right)=a\left(\boldsymbol{\vartheta}_{h}-\pi_{h}(\boldsymbol{\vartheta}),\right. & \left.\boldsymbol{\vartheta}_{h}^{\prime}\right)+b_{\alpha}\left(\boldsymbol{\vartheta}_{h}-\pi_{h}(\boldsymbol{\vartheta}), \boldsymbol{\vartheta}_{h}^{\prime}\right) \\
& +k\left(\boldsymbol{\vartheta}_{h}-\pi_{h}(\boldsymbol{\vartheta}), \boldsymbol{\vartheta}_{h}^{\prime}\right)+b_{\alpha}\left(\pi_{h}(\boldsymbol{\vartheta})-\boldsymbol{\vartheta}, \boldsymbol{\vartheta}_{h}^{\prime}\right),
\end{aligned}
$$

thanks to the definition of $\pi_{h}$. Choosing $\boldsymbol{\vartheta}_{h}^{\prime}=\boldsymbol{\vartheta}_{h}-\pi_{h}(\boldsymbol{\vartheta})$, we obtain:

$$
\begin{aligned}
m\left(\partial _ { t } \left(\boldsymbol{\vartheta}_{h}-\right.\right. & \left.\left.\pi_{h}(\boldsymbol{\vartheta})\right), \boldsymbol{\vartheta}_{h}-\pi_{h}(\boldsymbol{\vartheta})\right)=a\left(\boldsymbol{\vartheta}_{h}-\pi_{h}(\boldsymbol{\vartheta}), \boldsymbol{\vartheta}_{h}-\pi_{h}(\boldsymbol{\vartheta})\right) \\
+b_{\alpha}\left(\boldsymbol{\vartheta}_{h}-\pi_{h}(\boldsymbol{\vartheta}), \boldsymbol{\vartheta}_{h}-\pi_{h}(\boldsymbol{\vartheta})\right)+k\left(\boldsymbol{\vartheta}_{h}-\right. & \left.\pi_{h}(\boldsymbol{\vartheta}), \boldsymbol{\vartheta}_{h}-\pi_{h}(\boldsymbol{\vartheta})\right) \\
& +b_{\alpha}\left(\pi_{h}(\boldsymbol{\vartheta})-\boldsymbol{\vartheta}, \boldsymbol{\vartheta}_{h}-\pi_{h}(\boldsymbol{\vartheta})\right)
\end{aligned}
$$

From Lemma 4.2 and 4.3 , we deduce the inequality:

$$
m\left(\partial_{t}\left(\boldsymbol{\vartheta}_{h}-\pi_{h}(\boldsymbol{\vartheta})\right), \boldsymbol{\vartheta}_{h}-\pi_{h}(\boldsymbol{\vartheta})\right) \lesssim\left\|\boldsymbol{\vartheta}_{h}-\pi_{h}(\boldsymbol{\vartheta})\right\|^{2}+\eta h^{-1}\left\|\boldsymbol{\vartheta}-\pi_{h}(\boldsymbol{\vartheta})\right\|\left\|\boldsymbol{\vartheta}_{h}-\pi_{h}(\boldsymbol{\vartheta})\right\|
$$

Using the classical approximation estimate 4.7) and equivalence of norms, we obtain

$$
m\left(\partial_{t}\left(\boldsymbol{\vartheta}_{h}-\pi_{h}(\boldsymbol{\vartheta})\right), \boldsymbol{\vartheta}_{h}-\pi_{h}(\boldsymbol{\vartheta})\right) \lesssim\left\|\boldsymbol{\vartheta}_{h}-\pi_{h}(\boldsymbol{\vartheta})\right\|_{\Lambda}^{2}+\eta h^{\min (s, k)}|\boldsymbol{\vartheta}|_{\mathbf{H}^{s}(\Omega)^{N}}\left\|\boldsymbol{\vartheta}_{h}-\pi_{h}(\boldsymbol{\vartheta})\right\|_{\Lambda}
$$

This implies that

$$
\frac{d}{d t} \tilde{\mathcal{E}}_{h}(t) \lesssim\left\|\boldsymbol{\vartheta}_{h}-\pi_{h}(\boldsymbol{\vartheta})\right\|_{\Lambda}^{2}+\eta h^{\min (s, k)}|\boldsymbol{\vartheta}|_{\mathbf{H}^{s}(\Omega)^{N}}\left\|\boldsymbol{\vartheta}_{h}-\pi_{h}(\boldsymbol{\vartheta})\right\|_{\Lambda} .
$$

with $\tilde{\mathcal{E}}_{h}:=\frac{1}{2}\left\|\boldsymbol{\vartheta}_{h}-\pi_{h}(\boldsymbol{\vartheta})\right\|_{\Lambda}^{2}$. In other words, there exists $C>0$ such that

$$
\frac{d}{d t} \tilde{\mathcal{E}}_{h}(t) \leq 2 C \tilde{\mathcal{E}}_{h}(t)+2 C \eta h^{\min (s, k)}|\boldsymbol{\vartheta}|_{\mathbf{H}^{s}(\Omega)^{N}} \sqrt{\tilde{\mathcal{E}}_{h}(t)},
$$

or

$$
\frac{d}{d t}\left(\exp (-2 C t) \tilde{\mathcal{E}}_{h}(t)\right) \leq 2 C \eta h^{\min (s, k)} \exp (-C t)|\boldsymbol{\vartheta}|_{\mathbf{H}^{s}(\Omega)^{N}} \sqrt{\exp (-2 C t) \tilde{\mathcal{E}_{h}}(t)} .
$$

This gives

$$
\frac{d}{d t}\left(\sqrt{\exp (-2 C t) \tilde{\mathcal{E}}_{h}(t)}\right) \leq C \eta h^{\min (s, k)} \exp (-C t)|\boldsymbol{\vartheta}|_{\mathbf{H}^{s}(\Omega)^{N}},
$$

and

$$
\sqrt{\tilde{\mathcal{E}}_{h}(t)} \lesssim \exp (C t) \sqrt{\tilde{\mathcal{E}}_{h}(0)}+\eta h^{\min (s, k)}(\exp (C t)-1) \max _{[0, T]}\left(|\boldsymbol{\vartheta}|_{\mathbf{H}^{s}(\Omega)^{N}}\right)
$$

Combining this estimate with an approximation estimate and the triangle inequality, we deduce that the error $\left\|\boldsymbol{\vartheta}-\boldsymbol{\vartheta}_{h}\right\|$ is of order $O\left(h^{\min (s, k)}\right)$, if $\tilde{\mathcal{E}}_{h}(0)=O\left(h^{\min (s, k)}\right)$.

This concludes the study of the semi-convergence of the scheme. Let $\boldsymbol{\vartheta}$ be the solution of (3.4) and suppose that there exists $s \geq 1$ such that $\boldsymbol{\vartheta} \in \mathcal{C}^{0}\left(0, T,\left(\mathbf{H}^{s}(\Omega)\right)^{N}\right)$, then if $\boldsymbol{\vartheta}_{h}$ is the solution of 4.5 and $\left\|\boldsymbol{\vartheta}_{h}(0)-\pi_{h}(\boldsymbol{\vartheta})(0)\right\| \lesssim h^{\min (s, k)}$, one has:

$$
\left\|\boldsymbol{\vartheta}-\boldsymbol{\vartheta}_{h}\right\| \lesssim h^{\min (s, k)}\|\boldsymbol{\vartheta}\|_{\mathcal{C}^{0}\left(0, T,\left(\mathbf{H}^{s}(\Omega)\right)\right)^{N}} .
$$


This result holds for any non negative value of $\alpha$. However, if $\alpha>0$, we can improve the rate of convergence by exploiting the dissipative terms introduced by the fluxes. We rather writes 4.8 as:

$$
\begin{aligned}
m\left(\partial_{t}\left(\boldsymbol{\vartheta}_{h}-\pi_{h}(\boldsymbol{\vartheta})\right), \boldsymbol{\vartheta}_{h}-\pi_{h}(\boldsymbol{\vartheta})\right) \lesssim-\alpha\left|\boldsymbol{\vartheta}_{h}-\pi_{h}(\boldsymbol{\vartheta})\right|_{S}^{2}+\left\|\boldsymbol{\vartheta}_{h}-\pi_{h}(\boldsymbol{\vartheta})\right\|^{2} \\
+\eta h^{-\frac{1}{2}}\left\|\boldsymbol{\vartheta}-\pi_{h}(\boldsymbol{\vartheta})\right\|\left|\boldsymbol{\vartheta}_{h}-\pi_{h}(\boldsymbol{\vartheta})\right|_{S}
\end{aligned}
$$

Thus

$\frac{d}{d t} \tilde{\mathcal{E}}_{h}(t) \lesssim-\alpha\left|\boldsymbol{\vartheta}_{h}-\pi_{h}(\boldsymbol{\vartheta})\right|_{S}^{2}+\left\|\boldsymbol{\vartheta}_{h}-\pi_{h}(\boldsymbol{\vartheta})\right\|_{\Lambda}^{2}+\eta h^{\min (s, k)+\frac{1}{2}}|\boldsymbol{\vartheta}|_{\mathbf{H}^{s}(\Omega)^{N}}\left|\boldsymbol{\vartheta}_{h}-\pi_{h}(\boldsymbol{\vartheta})\right|_{S}$

We deduce

$$
\begin{aligned}
\frac{d}{d t} \tilde{\mathcal{E}}_{h}(t) & \lesssim-\frac{\alpha}{2}\left|\boldsymbol{\vartheta}_{h}-\pi_{h}(\boldsymbol{\vartheta})\right|_{S}^{2}+\left\|\boldsymbol{\vartheta}_{h}-\pi_{h}(\boldsymbol{\vartheta})\right\|_{\Lambda}^{2}+\frac{\eta^{2}}{2 \alpha} h^{2 m i n(s, k)+1}|\boldsymbol{\vartheta}|_{\mathbf{H}^{s}(\Omega)^{N}}^{2} \\
& \lesssim\left\|\boldsymbol{\vartheta}_{h}-\pi_{h}(\boldsymbol{\vartheta})\right\|_{\Lambda}^{2}+\frac{\eta^{2}}{2 \alpha} h^{2 m i n(s, k)+1}|\boldsymbol{\vartheta}|_{\mathbf{H}^{s}(\Omega)^{N}}^{2},
\end{aligned}
$$

that yields, thanks to a Grönwall type inequality a $O\left(h^{\min (s, k)+\frac{1}{2}}\right)$ convergence rate, if $\tilde{\mathcal{E}}_{h}(0)=O\left(h^{\min (s, k)+\frac{1}{2}}\right)$ and $\alpha>0$.

5. Fully discrete scheme. We are interested in deriving a fully discrete scheme for the considered problem. The semi-discretization relies on a arbitrarily high order scheme, so that we would be interested in a high order time scheme. We propose to use an explicit Runge Kutta (RK) scheme of order 4. Explicit schemes coupled to the discontinuous Galerkin framework proposed above, allow us tu exploit the block diagonal structure of the mass matrix leading to no large matrix inversion. This, as usual, will result in a constraint in the time step in terms of a CFL condition. Let us also mention that the implicit counterpart of RK schemes coupled to a discontinuous Galerkin framework have been recently studied in the context of linear Maxwell's equations in [HP15].

We first introduce all the notations, then will present a stability analysis and prove that the scheme is stable under a CFL condition. We will also see that this proof could extend to the fully discrete convergence of the scheme.

The approach chosen here is inspired from the key ideas of [LT98] and follows closely [BEF10]. In [LT98, among other results, the stability and convergence of explicit Runge Kutta (RK) schemes up to order 4 and in a ODE context is presented via the use of an energy principle. Their stability analysis strongly rely on one hypothesis of (negative) coercivity of the operator that we do not have here. However we can explicitly take into account for the semi-discretization of the scheme and our discrete DG operator has an dissipative nature that we will exploit as in BEF10. In this reference, the convergence analysis has been developed for RK2 and RK3 schemes. We develop the extension of this stability analysis to a compact perturbed problem and for the 4 th order Runge Kutta scheme, in the energy norm $\|\cdot\|_{\Lambda}$. The study of this scheme has moreover to be written carefully since a straightforward analysis (as a direct extension of [BEF10]) would lead to a more restrictive CFL condition compared to what would have been expected from the analysis of the RK2 and RK3 schemes.

We thus propose here to extensively detail the energy stability analysis. This is the first and essential step towards a complete convergence proof. We choose not 
to detail the latter to ease the reading of an unused numerical analysis reader, since the computations for the stability analysis are already quite fastidious. This first step reveals the key ingredients for a whole convergence proof relying on error energy principles and consistency type results, and we chose to rather sketch it since it would then readily follow a combination of e.g. BEF10, LS13, and the semi-discrete convergence.

5.1. Notations. We introduce the following notations. Let us define the operators $\mathfrak{L}_{h}$ by:

$$
\mathfrak{L}_{h}: \mathbb{V}_{h} \rightarrow \mathbb{V}_{h},
$$

with for all $(\boldsymbol{\varsigma}, \boldsymbol{\xi}) \in \mathbb{V}_{h} \times \mathbb{V}_{h}$ :

$$
\left(\mathfrak{L}_{h} \boldsymbol{\varsigma}, \boldsymbol{\xi}\right)=a(\boldsymbol{\varsigma}, \boldsymbol{\xi})+b_{\alpha}(\boldsymbol{\varsigma}, \boldsymbol{\xi}),
$$

and $\mathfrak{K}_{h}$ defined by:

$$
\mathfrak{K}_{h}: \mathbb{V}_{h} \rightarrow \mathbb{V}_{h},
$$

with for all $\left(\mathfrak{K}_{h} \boldsymbol{\varsigma}, \boldsymbol{\xi}\right)=k(\boldsymbol{\varsigma}, \boldsymbol{\xi})$, and finally:

$$
\mathfrak{D}_{h}:=\mathfrak{L}_{h}+\mathfrak{K}_{h} .
$$

The semi-discrete scheme can thus be rewritten as: Find $\boldsymbol{\vartheta}_{h} \in \mathbb{V}_{h}$ such that:

$$
\Lambda \frac{\partial \vartheta_{h}}{\partial t}=\mathfrak{D}_{h}\left(\boldsymbol{\vartheta}_{h}\right)
$$

For $M \in \mathbb{N}^{*}$, we denote $\Delta t:=\frac{T}{M}$ and $\left(t_{n}\right)_{n \in[0, M]}$ a uniform subdivision of $[0, T]$ of step $\Delta t$.

In this section, we denote $\alpha \lesssim \beta$, to express that there exists $C>0$ (independent of the mesh size $h$ and time step $\Delta t$ ) such that $\alpha \leq C \beta$.

From Lemma 4.3 , it follows

$$
\begin{gathered}
\left\|\left|\mathfrak{L}_{h}\right|\right\| \lesssim \eta h^{-1}, \\
\left\|\left|\mathfrak{K}_{h}\right|\right\| \lesssim 1 .
\end{gathered}
$$

We propose to study a Runge-Kutta discretization, namely RK4. It can be easily written as: $\forall n \in[1, M]$, find $\boldsymbol{\vartheta}_{h}^{n} \in \mathbb{V}_{h}$ such that:

$$
\left\{\begin{array}{l}
\boldsymbol{\vartheta}_{h}^{n+1}=\boldsymbol{\vartheta}_{h}^{n}+\sum_{i=1}^{4} \frac{1}{i !} \Delta t^{i}\left(\Lambda^{-1} \mathfrak{D}_{h}\right)^{i}\left(\boldsymbol{\vartheta}_{h}^{n}\right), \forall n \in[0, M-1], \\
\boldsymbol{\vartheta}_{h}^{0} \text { given in } \mathbb{V}_{h} .
\end{array}\right.
$$

The scheme can also be expressed in its four step version as follows: $\forall n \in[0, M-1]$,

$$
\begin{aligned}
\boldsymbol{w}^{n} & =\boldsymbol{\vartheta}_{h}^{n}+\Delta t \Lambda^{-1} \mathfrak{D}_{h}\left(\boldsymbol{\vartheta}_{h}^{n}\right), \\
\boldsymbol{y}^{n} & =\frac{1}{2}\left(\boldsymbol{\vartheta}_{h}^{n}+\boldsymbol{w}^{n}\right)+\frac{1}{2} \Delta t \Lambda^{-1} \mathfrak{D}_{h}\left(\boldsymbol{w}^{n}\right), \\
\boldsymbol{z}^{n} & =\frac{1}{3}\left(\boldsymbol{\vartheta}_{h}^{n}+\boldsymbol{w}^{n}+\boldsymbol{y}^{n}\right)+\frac{1}{3} \Delta t \Lambda^{-1} \mathfrak{D}_{h}\left(\boldsymbol{y}^{n}\right), \\
\boldsymbol{\vartheta}_{h}^{n+1} & =\frac{1}{4}\left(\boldsymbol{\vartheta}_{h}^{n}+\boldsymbol{w}^{n}+\boldsymbol{y}^{n}+\boldsymbol{z}^{n}\right)+\frac{1}{4} \Delta t \Lambda^{-1} \mathfrak{D}_{h}\left(\boldsymbol{z}^{n}\right) .
\end{aligned}
$$


We deduce that $\forall n \in[0, M-1]$,

$$
\begin{aligned}
\boldsymbol{y}^{n}-\boldsymbol{w}^{n} & =-\frac{1}{2} \Delta t \Lambda^{-1} \mathfrak{D}_{h}\left(\boldsymbol{\vartheta}_{h}^{n}-\boldsymbol{w}^{n}\right), \\
\boldsymbol{z}^{n}-\boldsymbol{y}^{n} & =-\frac{1}{3} \Delta t \Lambda^{-1} \mathfrak{D}_{h}\left(\boldsymbol{w}^{n}-\boldsymbol{y}^{n}\right), \\
\boldsymbol{\vartheta}_{h}^{n+1}-\boldsymbol{z}^{n} & =-\frac{1}{4} \Delta t \Lambda^{-1} \mathfrak{D}_{h}\left(\boldsymbol{y}^{n}-\boldsymbol{z}^{n}\right) .
\end{aligned}
$$

REMARK 5. If one would only consider the three first steps to compute $\boldsymbol{\vartheta}_{h}^{n+1}$, one ends up with the Runge Kutta 3 scheme.

5.2. Stability of the fully discrete scheme. In this section, we prove the stability of the fully discrete scheme.

Proposition 5.1. Let $\alpha \in] 0,1]$. Under a $\frac{4}{3}$-CFL condition, i.e. $\Delta t \lesssim h^{\frac{4}{3}}$, the scheme is stable in finite time, in the sense that there exists $C>0$ (independent of $h$ and $\Delta t$ ) such that:

$$
\max _{n \in[0, M]}\left\|\boldsymbol{\vartheta}_{h}^{n}\right\|_{\Lambda}^{2} \leq \exp (C T)\left\|\boldsymbol{\vartheta}_{h}^{0}\right\|_{\Lambda}^{2}
$$

Proof. Let us study the variation of the energy defined by $\mathcal{E}_{h}^{n}=\frac{1}{2}\left\|\boldsymbol{\vartheta}_{h}^{n}\right\|_{\Lambda}^{2}$ in discrete time. One finds first:

$$
\begin{aligned}
\frac{1}{2}\left\|\boldsymbol{\vartheta}_{h}^{n+1}\right\|_{\Lambda}^{2}-\frac{1}{2}\left\|\boldsymbol{\vartheta}_{h}^{n+1}-\boldsymbol{z}^{n}\right\|_{\Lambda}^{2}-\frac{1}{2}\left\|\boldsymbol{\vartheta}_{h}^{n}\right\|_{\Lambda}^{2} & =\frac{1}{2}\left(2 \boldsymbol{\vartheta}_{h}^{n+1}-\boldsymbol{z}^{n}, \boldsymbol{z}^{n}\right)_{\Lambda}-\frac{1}{2}\left\|\boldsymbol{\vartheta}_{h}^{n}\right\|_{\Lambda}^{2} \\
& =\left(\boldsymbol{\vartheta}_{h}^{n+1}-\frac{1}{2} \boldsymbol{z}^{n}, \boldsymbol{z}^{n}\right)_{\Lambda}-\frac{1}{2}\left\|\boldsymbol{\vartheta}_{h}^{n}\right\|_{\Lambda}^{2} \\
& =\frac{1}{2}\left\|\boldsymbol{z}^{n}\right\|_{\Lambda}^{2}+\left(\boldsymbol{\vartheta}_{h}^{n+1}-\boldsymbol{z}^{n}, \boldsymbol{z}^{n}\right)_{\Lambda}-\frac{1}{2}\left\|\boldsymbol{\vartheta}_{h}^{n}\right\|_{\Lambda}^{2} .
\end{aligned}
$$

Furthermore, we can prove after some manipulations that:

$$
\left\|\boldsymbol{y}^{n}\right\|_{\Lambda}^{2}-\left\|\boldsymbol{\vartheta}_{h}^{n}\right\|_{\Lambda}^{2}=\left\|\boldsymbol{y}^{n}-\boldsymbol{w}^{n}\right\|_{\Lambda}^{2}+\Delta t\left(\mathfrak{D}_{h}\left(\boldsymbol{\vartheta}_{h}^{n}\right), \boldsymbol{\vartheta}_{h}^{n}\right)+\Delta t\left(\mathfrak{D}_{h}\left(\boldsymbol{w}^{n}\right), \boldsymbol{w}^{n}\right)
$$

and

$$
\begin{aligned}
\frac{1}{2}\left\|\boldsymbol{z}^{n}\right\|_{\Lambda}^{2}-\frac{1}{2}\left\|\boldsymbol{\vartheta}_{h}^{n}\right\|_{\Lambda}^{2}=\frac{1}{2} \Delta t\left(\mathfrak{D}_{h}\left(\boldsymbol{\vartheta}_{h}^{n}\right), \boldsymbol{\vartheta}_{h}^{n}\right)+\frac{1}{6} \Delta t\left(\mathfrak{D}_{h}\left(\boldsymbol{w}^{n}\right), \boldsymbol{w}^{n}\right) \\
+\frac{1}{3} \Delta t\left(\mathfrak{D}_{h}\left(\boldsymbol{y}^{n}\right), \boldsymbol{y}^{n}\right)-\frac{1}{6}\left\|\boldsymbol{y}^{n}-\boldsymbol{w}^{n}\right\|_{\Lambda}^{2} \\
\quad-\frac{1}{6} \Delta t\left(\mathfrak{D}_{h}\left(\boldsymbol{w}^{n}-\boldsymbol{\vartheta}_{h}^{n}\right), \boldsymbol{w}^{n}-\boldsymbol{\vartheta}_{h}^{n}\right)+\frac{1}{2}\left\|\boldsymbol{z}^{n}-\boldsymbol{y}^{n}\right\|_{\Lambda}^{2},
\end{aligned}
$$

REMARK 6. Equations (5.11) (resp. (5.12) corresponds to the energy principle for the RK2 (resp. RK3) scheme. Since this mimics exactly the results in [BEF10], we choose not to detail it.

Furthermore

$$
\begin{aligned}
\left(\boldsymbol{\vartheta}_{h}^{n+1}-\boldsymbol{z}^{n}, \boldsymbol{z}^{n}\right)_{\Lambda} & =-\frac{1}{4} \Delta t\left(\mathfrak{D}_{h}\left(\boldsymbol{y}^{n}-\boldsymbol{z}^{n}\right), \boldsymbol{z}^{n}\right) \\
& =\frac{1}{4} \Delta t\left(\mathfrak{D}_{h}\left(\boldsymbol{z}^{n}\right), \boldsymbol{z}^{n}\right)-\frac{1}{4} \Delta t\left(\mathfrak{D}_{h}\left(\boldsymbol{y}^{n}\right), \boldsymbol{z}^{n}\right)
\end{aligned}
$$


Thus:

$$
\begin{aligned}
\frac{1}{2}\left\|\boldsymbol{\vartheta}_{h}^{n+1}\right\|_{\Lambda}^{2}-\frac{1}{2}\left\|\boldsymbol{\vartheta}_{h}^{n}\right\|_{\Lambda}^{2}= & \frac{1}{2} \Delta t\left(\mathfrak{D}_{h}\left(\boldsymbol{\vartheta}_{h}^{n}\right), \boldsymbol{\vartheta}_{h}^{n}\right)+\frac{1}{6} \Delta t\left(\mathfrak{D}_{h}\left(\boldsymbol{w}^{n}\right), \boldsymbol{w}^{n}\right)+\frac{1}{3} \Delta t\left(\mathfrak{D}_{h}\left(\boldsymbol{y}^{n}\right), \boldsymbol{y}^{n}\right) \\
& -\frac{1}{6}\left\|\boldsymbol{y}^{n}-\boldsymbol{w}^{n}\right\|_{\Lambda}^{2}-\frac{1}{6} \Delta t\left(\mathfrak{D}_{h}\left(\boldsymbol{w}^{n}-\boldsymbol{\vartheta}_{h}^{n}\right), \boldsymbol{w}^{n}-\boldsymbol{\vartheta}_{h}^{n}\right)+\frac{1}{2}\left\|\boldsymbol{z}^{n}-\boldsymbol{y}^{n}\right\|_{\Lambda}^{2} \\
& +\frac{1}{4} \Delta t\left(\mathfrak{D}_{h}\left(\boldsymbol{z}^{n}\right), \boldsymbol{z}^{n}\right)-\frac{1}{4} \Delta t\left(\mathfrak{D}_{h}\left(\boldsymbol{y}^{n}\right), \boldsymbol{z}^{n}\right)+\frac{1}{2}\left\|\boldsymbol{\vartheta}_{h}^{n+1}-\boldsymbol{z}^{n}\right\|_{\Lambda}^{2} .
\end{aligned}
$$

But:

$$
-\frac{1}{4}\left(\mathfrak{D}_{h}\left(\boldsymbol{y}^{n}\right), \boldsymbol{z}^{n}\right)=-\frac{1}{4}\left(\mathfrak{D}_{h}\left(\boldsymbol{y}^{n}\right), \boldsymbol{z}^{n}-\boldsymbol{y}^{n}\right)-\frac{1}{4}\left(\mathfrak{D}_{h}\left(\boldsymbol{y}^{n}\right), \boldsymbol{y}^{n}\right) .
$$

This yields:

$$
\begin{aligned}
\frac{1}{2}\left\|\boldsymbol{\vartheta}_{h}^{n+1}\right\|_{\Lambda}^{2}-\frac{1}{2}\left\|\boldsymbol{\vartheta}_{h}^{n}\right\|_{\Lambda}^{2}= & \frac{1}{2} \Delta t\left(\mathfrak{D}_{h}\left(\boldsymbol{\vartheta}_{h}^{n}\right), \boldsymbol{\vartheta}_{h}^{n}\right)+\frac{1}{6} \Delta t\left(\mathfrak{D}_{h}\left(\boldsymbol{w}^{n}\right), \boldsymbol{w}^{n}\right)+\frac{1}{12} \Delta t\left(\mathfrak{D}_{h}\left(\boldsymbol{y}^{n}\right), \boldsymbol{y}^{n}\right)-\frac{1}{6}\left\|\boldsymbol{y}^{n}-\boldsymbol{w}^{n}\right\|_{\Lambda}^{2} \\
& -\frac{1}{6} \Delta t\left(\mathfrak{D}_{h}\left(\boldsymbol{w}^{n}-\boldsymbol{\vartheta}_{h}^{n}\right), \boldsymbol{w}^{n}-\boldsymbol{\vartheta}_{h}^{n}\right)+\frac{1}{2}\left\|\boldsymbol{z}^{n}-\boldsymbol{y}^{n}\right\|_{\Lambda}^{2}+\frac{1}{4} \Delta t\left(\mathfrak{D}_{h}\left(\boldsymbol{z}^{n}\right), \boldsymbol{z}^{n}\right) \\
& -\frac{1}{4} \Delta t\left(\mathfrak{D}_{h}\left(\boldsymbol{y}^{n}\right), \boldsymbol{z}^{n}-\boldsymbol{y}^{n}\right)+\frac{1}{2}\left\|\boldsymbol{\vartheta}_{h}^{n+1}-\boldsymbol{z}^{n}\right\|_{\Lambda}^{2} .
\end{aligned}
$$

Furthermore,

$$
\begin{aligned}
\frac{1}{2}\left\|\boldsymbol{z}^{n}-\boldsymbol{y}^{n}\right\|_{\Lambda}^{2}-\frac{1}{4} \Delta t\left(\mathfrak{D}_{h}\left(\boldsymbol{y}^{n}\right), \boldsymbol{z}^{n}-\boldsymbol{y}^{n}\right)= & \frac{1}{2}\left(\boldsymbol{z}^{n}-\boldsymbol{y}^{n}, \Lambda \boldsymbol{z}^{n}-\Lambda \boldsymbol{y}^{n}-\frac{1}{2} \Delta t \mathfrak{D}_{h}\left(\boldsymbol{y}^{n}\right)\right) \\
= & \frac{1}{2}\left(\boldsymbol{z}^{n}-\boldsymbol{y}^{n},-\frac{1}{2}\left(\Lambda \boldsymbol{z}^{n}-\Lambda \boldsymbol{y}^{n}\right)+\frac{3}{2}\left(\Lambda \boldsymbol{z}^{n}-\Lambda \boldsymbol{y}^{n}\right)-\frac{1}{2} \Delta t \mathfrak{D}_{h}\left(\boldsymbol{y}^{n}\right)\right) \\
= & -\frac{1}{4}\left\|\boldsymbol{z}^{n}-\boldsymbol{y}^{n}\right\|_{\Lambda}^{2}+\frac{1}{2}\left(\boldsymbol{z}^{n}-\boldsymbol{y}^{n},-\frac{1}{2} \Delta t \mathfrak{D}_{h}\left(\boldsymbol{w}^{n}-\boldsymbol{y}^{n}\right)-\frac{1}{2} \Delta t \mathfrak{D}_{h}\left(\boldsymbol{y}^{n}\right)\right) \\
= & -\frac{1}{4}\left\|\boldsymbol{z}^{n}-\boldsymbol{y}^{n}\right\|_{\Lambda}^{2}+\frac{1}{2}\left(\boldsymbol{z}^{n}-\boldsymbol{y}^{n},-\frac{1}{2} \Delta t \mathfrak{D}_{h}\left(\boldsymbol{w}^{n}\right)\right) \\
= & -\frac{1}{4}\left\|\boldsymbol{z}^{n}-\boldsymbol{y}^{n}\right\|_{\Lambda}^{2}+\frac{1}{2}\left(\boldsymbol{z}^{n}-\boldsymbol{y}^{n}, \frac{1}{2}\left(\Lambda \boldsymbol{\vartheta}_{h}^{n}-\Lambda \boldsymbol{w}^{n}\right)+\left(\Lambda \boldsymbol{w}^{n}-\Lambda \boldsymbol{y}^{n}\right)\right) \\
= & -\frac{1}{4}\left\|\boldsymbol{z}^{n}-\boldsymbol{y}^{n}\right\|_{\Lambda}^{2}+\frac{1}{12}\left(-\Delta t \mathfrak{D}_{h}\left(\boldsymbol{w}^{n}-\boldsymbol{y}^{n}\right), \boldsymbol{\vartheta}_{h}^{n}-\boldsymbol{w}^{n}\right) \\
& +\frac{1}{6}\left(-\Delta t \mathfrak{D}_{h}\left(\boldsymbol{w}^{n}-\boldsymbol{y}^{n}\right),\left(\boldsymbol{w}^{n}-\boldsymbol{y}^{n}\right)\right) .
\end{aligned}
$$

The second term of the right hand side can be written as:

$$
\begin{aligned}
-\frac{1}{12} \Delta t\left(\mathfrak{D}_{h}\left(\boldsymbol{w}^{n}-\boldsymbol{y}^{n}\right), \boldsymbol{\vartheta}_{h}^{n}-\boldsymbol{w}^{n}\right) & =-\frac{1}{24} \Delta t^{2}\left(\left(\Lambda^{-1} \mathfrak{D}_{h}\right) \circ \mathfrak{D}_{h}\left(\boldsymbol{\vartheta}_{h}^{n}-\boldsymbol{w}^{n}\right), \boldsymbol{\vartheta}_{h}^{n}-\boldsymbol{w}^{n}\right) \\
& =-\frac{1}{24} \Delta t^{4}\left(\left(\Lambda^{-1} \mathfrak{D}_{h}\right) \circ\left(\Lambda^{-1} \mathfrak{D}_{h}\right) \circ \mathfrak{D}_{h}\left(\boldsymbol{\vartheta}_{h}^{n}\right),\left(\Lambda^{-1} \mathfrak{D}_{h}\right)\left(\boldsymbol{\vartheta}_{h}^{n}\right)\right),
\end{aligned}
$$


using (5.4). We are able to summarize the results in an energy equality as follows:

$$
\begin{aligned}
\frac{1}{2}\left\|\boldsymbol{\vartheta}_{h}^{n+1}\right\|_{\Lambda}^{2}-\frac{1}{2}\left\|\boldsymbol{\vartheta}_{h}^{n}\right\|_{\Lambda}^{2} & =\frac{1}{2} \Delta t\left(\mathfrak{D}_{h}\left(\boldsymbol{\vartheta}_{h}^{n}\right), \boldsymbol{\vartheta}_{h}^{n}\right)+\frac{1}{6} \Delta t\left(\mathfrak{D}_{h}\left(\boldsymbol{w}^{n}\right), \boldsymbol{w}^{n}\right) \\
& +\frac{1}{12} \Delta t\left(\mathfrak{D}_{h}\left(\boldsymbol{y}^{n}\right), \boldsymbol{y}^{n}\right)-\frac{1}{6}\left\|\boldsymbol{y}^{n}-\boldsymbol{w}^{n}\right\|_{\Lambda}^{2}-\frac{1}{6} \Delta t\left(\mathfrak{D}_{h}\left(\boldsymbol{w}^{n}-\boldsymbol{\vartheta}_{h}^{n}\right), \boldsymbol{w}^{n}-\boldsymbol{\vartheta}_{h}^{n}\right) \\
& +\frac{1}{4} \Delta t\left(\mathfrak{D}_{h}\left(\boldsymbol{z}^{n}\right), \boldsymbol{z}^{n}\right)+\frac{1}{2}\left\|\boldsymbol{\vartheta}_{h}^{n+1}-\boldsymbol{z}^{n}\right\|_{\Lambda}^{2}-\frac{1}{4}\left\|\boldsymbol{z}^{n}-\boldsymbol{y}^{n}\right\|_{\Lambda}^{2} \\
& -\frac{1}{24} \Delta t^{4}\left(\left(\Lambda^{-1} \mathfrak{D}_{h}\right) \circ\left(\Lambda^{-1} \mathfrak{D}_{h}\right) \circ \mathfrak{D}_{h}\left(\boldsymbol{\vartheta}_{h}^{n}\right),\left(\Lambda^{-1} \mathfrak{D}_{h}\right)\left(\boldsymbol{\vartheta}_{h}^{n}\right)\right) \\
& +\frac{1}{6}\left(-\Delta t \mathfrak{D}_{h}\left(\boldsymbol{w}^{n}-\boldsymbol{y}^{n}\right),\left(\boldsymbol{w}^{n}-\boldsymbol{y}^{n}\right)\right)
\end{aligned}
$$

Furthermore, from the definition of $\mathfrak{D}_{h}, \mathfrak{L}_{h}$ and $\mathfrak{K}_{h}, \forall \boldsymbol{\vartheta}_{h} \in \mathbb{V}_{h}$ :

$$
\left(\mathfrak{L}_{h}\left(\boldsymbol{\vartheta}_{h}\right), \boldsymbol{\vartheta}_{h}\right)=a\left(\boldsymbol{\vartheta}_{h}, \boldsymbol{\vartheta}_{h}\right)+b_{\alpha}\left(\boldsymbol{\vartheta}_{h}, \boldsymbol{\vartheta}_{h}\right)=-\alpha\left(\frac{1}{Y}\left\|\llbracket \boldsymbol{\vartheta}_{1} \rrbracket\right\|_{\mathcal{F}_{h}^{\text {int }}}^{2}+\frac{1}{Z}\left\|\llbracket \llbracket \boldsymbol{\vartheta}_{2} \rrbracket\right\|_{\mathcal{F}_{h}^{\text {int }}}^{2}\right) .
$$

so that:

$$
\left(\mathfrak{D}_{h}\left(\boldsymbol{\vartheta}_{h}\right), \boldsymbol{\vartheta}_{h}\right)=-\alpha\left(\frac{1}{\bar{Y}}\left\|\llbracket \boldsymbol{\vartheta}_{1} \rrbracket\right\|_{\mathcal{F}_{h}^{\text {int }}}^{2}+\frac{1}{\bar{Z}}\left\|\llbracket \llbracket \boldsymbol{\vartheta}_{2} \rrbracket\right\|_{\mathcal{F}_{h}^{\text {int }}}^{2}\right)+\left(\mathfrak{K}_{h}\left(\boldsymbol{\vartheta}_{h}\right), \boldsymbol{\vartheta}_{h}\right)
$$

Recall that we have defined for $\boldsymbol{\vartheta}_{h} \in \mathbb{V}_{h},\left|\boldsymbol{\vartheta}_{h}\right|_{S}^{2}:=\frac{1}{\bar{Y}}\left\|\llbracket \boldsymbol{\vartheta}_{1} \rrbracket\right\|_{\mathcal{F}_{h}^{\text {int }}}^{2}+\frac{1}{\bar{Z}}\left\|\llbracket \boldsymbol{\vartheta}_{2} \rrbracket\right\|_{\mathcal{F}_{h}^{\text {int }}}^{2}$.

Then (5.13) writes:

$$
\left(\mathfrak{D}_{h}\left(\boldsymbol{\vartheta}_{h}\right), \boldsymbol{\vartheta}_{h}\right)=-\alpha\left|\boldsymbol{\vartheta}_{h}\right|_{S}^{2}+\left(\mathfrak{K}_{h}\left(\boldsymbol{\vartheta}_{h}\right), \boldsymbol{\vartheta}_{h}\right) .
$$

The case $\mathfrak{K}_{h} \equiv 0$. As a first step, let us first imagine that $\mathfrak{K}_{h} \equiv 0$. In this particular case:

$$
\begin{aligned}
\frac{1}{2}\left\|\boldsymbol{\vartheta}_{h}^{n+1}\right\|_{\Lambda}^{2}-\frac{1}{2}\left\|\boldsymbol{\vartheta}_{h}^{n}\right\|_{\Lambda}^{2}= & -\frac{\alpha}{2} \Delta t\left|\boldsymbol{\vartheta}_{h}^{n}\right|_{S}^{2}-\frac{\alpha}{6} \Delta t\left|\boldsymbol{w}^{n}\right|_{S}^{2}-\frac{\alpha}{12} \Delta t\left|\boldsymbol{y}^{n}\right|_{S}^{2}-\frac{\alpha}{4} \Delta t\left|\boldsymbol{z}^{n}\right|_{S}^{2} \\
& +\frac{1}{6} \alpha \Delta t\left|\boldsymbol{w}^{n}-\boldsymbol{\vartheta}_{h}^{n}\right|_{S}^{2}+\frac{1}{6} \alpha \Delta t\left|\boldsymbol{w}^{n}-\boldsymbol{y}^{n}\right|_{S}^{2} \\
& -\frac{1}{6}\left\|\boldsymbol{y}^{n}-\boldsymbol{w}^{n}\right\|_{\Lambda}^{2}-\frac{1}{4}\left\|\boldsymbol{z}^{n}-\boldsymbol{y}^{n}\right\|_{\Lambda}^{2} \\
& -\frac{1}{24} \Delta t^{4}\left(\left(\Lambda^{-1} \mathfrak{D}_{h}\right) \circ\left(\Lambda^{-1} \mathfrak{D}_{h}\right) \circ \mathfrak{D}_{h}\left(\boldsymbol{\vartheta}_{h}^{n}\right),\left(\Lambda^{-1} \mathfrak{D}_{h}\right)\left(\boldsymbol{\vartheta}_{h}^{n}\right)\right) \\
& +\frac{1}{2}\left\|\boldsymbol{\vartheta}_{h}^{n+1}-\boldsymbol{z}^{n}\right\|_{\Lambda}^{2} .
\end{aligned}
$$

The only terms in 5.14 that could be (or are) non-negative are the last four terms: $R_{1}=\frac{1}{2}\left\|\boldsymbol{\vartheta}_{h}^{n+1}-\boldsymbol{z}^{n}\right\|_{\Lambda}^{2}, R_{2}=-\frac{1}{24} \Delta t^{4}\left(\left(\Lambda^{-1} \mathfrak{D}_{h}\right) \circ\left(\Lambda^{-1} \mathfrak{D}_{h}\right) \circ \mathfrak{D}_{h}\left(\boldsymbol{\vartheta}_{h}^{n}\right),\left(\Lambda^{-1} \mathfrak{D}_{h}\right)\left(\boldsymbol{\vartheta}_{h}^{n}\right)\right)$, $R_{3}=\frac{1}{6} \alpha \Delta t\left|\boldsymbol{w}^{n}-\boldsymbol{y}^{n}\right|_{S}^{2}$, and $R_{4}=\frac{1}{6} \alpha \Delta t\left|\boldsymbol{w}^{n}-\boldsymbol{\vartheta}_{h}^{n}\right|_{S}^{2}$.

REMARK 7. These terms are the antidissipative terms induced by the RungeKutta scheme, whereas the terms $-\frac{\alpha}{2} \Delta t\left|\boldsymbol{\vartheta}_{h}^{n}\right|_{S}^{2}-\frac{\alpha}{6} \Delta t\left|\boldsymbol{w}^{n}\right|_{S}^{2}-\frac{\alpha}{12} \Delta t\left|\boldsymbol{y}^{n}\right|_{S}^{2}-\frac{\alpha}{4} \Delta t\left|\boldsymbol{z}^{n}\right|_{S}^{2}$ are the dissipative terms introduced by the numerical flux. These dissipative terms will allow for compensating the antidissipative ones if $\alpha>0$. 
Study of $R_{1}$. Using (5.2):

$$
R_{1} \leq \frac{1}{32} \Delta t^{2}\left\|\mathfrak{L}_{h}\left(\boldsymbol{y}^{n}-\boldsymbol{z}^{n}\right)\right\|^{2} \leq \frac{1}{32}\left(\Delta t C h^{-1}\right)^{2}\left\|\boldsymbol{y}^{n}-\boldsymbol{z}^{n}\right\|_{\Lambda}^{2}
$$

Study of $R_{2}$. In a same manner:

$$
\left|R_{2}\right| \leq C \Delta t^{4} h^{-4}\left\|\vartheta_{h}^{n}\right\|_{\Lambda}^{2}
$$

Study of $R_{3}$. By inverse inequalities:

$$
R_{3} \leq \frac{1}{6} C \alpha \Delta t h^{-1}\left\|\boldsymbol{w}^{n}-\boldsymbol{y}^{n}\right\|_{\Lambda}^{2}
$$

Study of $R_{4}$. For $\nu, \tilde{\nu}, \hat{\nu}$ positive numbers given, if we would write directly, using Cauchy-Schwarz inequality several times :

$$
\begin{aligned}
R_{4} \leq & \alpha \frac{1}{6} \Delta t\left((1+\nu)\left|\boldsymbol{\vartheta}_{h}^{n}-\boldsymbol{y}^{n}\right|_{S}^{2}+\left(1+\nu^{-1}\right)\left|\boldsymbol{w}^{n}-\boldsymbol{y}^{n}\right|_{S}^{2}\right) \\
\leq & \alpha \frac{1}{6} \Delta t\left((1+\nu)(1+\hat{\nu})\left|\boldsymbol{\vartheta}_{h}^{n}-\boldsymbol{z}^{n}\right|_{S}^{2}+(1+\nu)\left(1+\hat{\nu}^{-1}\right)\left|\boldsymbol{z}^{n}-\boldsymbol{y}^{n}\right|_{S}^{2}+\left(1+\nu^{-1}\right)\left|\boldsymbol{w}^{n}-\boldsymbol{y}^{n}\right|_{S}^{2}\right) \\
\leq & \alpha \frac{1}{6} \Delta t\left((1+\nu)(1+\hat{\nu})(1+\tilde{\nu})\left|\boldsymbol{z}^{n}\right|_{S}^{2}+(1+\nu)(1+\hat{\nu})\left(1+\tilde{\nu}^{-1}\right)\left|\boldsymbol{\vartheta}_{h}^{n}\right|_{S}^{2}\right. \\
& \left.+(1+\nu)\left(1+\hat{\nu}^{-1}\right)\left|\boldsymbol{z}^{n}-\boldsymbol{y}^{n}\right|_{S}^{2}+\left(1+\nu^{-1}\right)\left|\boldsymbol{w}^{n}-\boldsymbol{y}^{n}\right|_{S}^{2}\right) \\
\leq & \alpha \frac{1}{6} \Delta t\left((1+\nu)(1+\hat{\nu})(1+\tilde{\nu})\left|\boldsymbol{z}^{n}\right|_{S}^{2}+(1+\nu)(1+\hat{\nu})\left(1+\tilde{\nu}^{-1}\right)\left|\boldsymbol{\vartheta}_{h}^{n}\right|_{S}^{2}\right) \\
& +\alpha \frac{1}{6}\left(C \Delta t h^{-1}(1+\nu)\left(1+\hat{\nu}^{-1}\right)\left\|\boldsymbol{z}^{n}-\boldsymbol{y}^{n}\right\|_{\Lambda}^{2}+C \Delta t h^{-1}\left(1+\nu^{-1}\right)\left\|\boldsymbol{w}^{n}-\boldsymbol{y}^{n}\right\|_{\Lambda}^{2}\right) .
\end{aligned}
$$

One would not find any values of $\nu, \tilde{\nu}, \hat{\nu}$, such that the positive terms $\left|\boldsymbol{z}^{n}\right|_{S}^{2}$, and $\left|\boldsymbol{\vartheta}_{h}^{n}\right|_{S}^{2}$ are compensated with the two terms $-\frac{\alpha}{2} \Delta t\left|\boldsymbol{\vartheta}_{h}^{n}\right|_{S}^{2}$ and $-\frac{\alpha}{4} \Delta t\left|\boldsymbol{z}^{n}\right|_{S}^{2}$. And without any further investigation, one could conclude that:

$$
\frac{1}{6} \Delta t\left|\boldsymbol{w}^{n}-\boldsymbol{\vartheta}_{h}^{n}\right|_{S}^{2} \leq \frac{1}{6} \Delta t^{3} h^{-3}\left|\boldsymbol{\vartheta}_{h}^{n}\right|_{S}^{2}
$$

Such an inequality would only lead to a $\frac{3}{2}$ CFL-condition. Instead, to have a better CFL condition, we propose to exploit the negative terms in $\left|\boldsymbol{w}^{n}\right|_{S}$. We write that:

$$
\frac{1}{6}\left|\boldsymbol{w}^{n}-\boldsymbol{\vartheta}_{h}^{n}\right|_{S}^{2}=\frac{1}{24}\left|\boldsymbol{w}^{n}-\boldsymbol{\vartheta}_{h}^{n}\right|_{S}^{2}+\frac{3}{24}\left|\boldsymbol{w}^{n}-\boldsymbol{\vartheta}_{h}^{n}\right|_{S}^{2}
$$

We then treat the two terms separately.

$$
\frac{1}{24}\left|\boldsymbol{w}^{n}-\boldsymbol{\vartheta}_{h}^{n}\right|_{S}^{2} \leq \frac{1}{12}\left|\boldsymbol{w}^{n}\right|_{S}^{2}+\frac{1}{12}\left|\boldsymbol{\vartheta}_{h}^{n}\right|_{S}^{2} .
$$


$\frac{1}{12}\left|\boldsymbol{w}^{n}\right|_{S}^{2}$ is compensated by the term $-\frac{1}{6}\left|\boldsymbol{w}^{n}\right|_{S}^{2}$, and the term $\frac{1}{12}\left|\boldsymbol{\vartheta}_{h}^{n}\right|_{S}^{2}$ by the corresponding negative one. For the second term, we write

$$
\begin{aligned}
\frac{3}{24} \alpha \Delta t\left|\boldsymbol{w}^{n}-\boldsymbol{\vartheta}_{h}^{n}\right|_{S}^{2} \leq & \alpha \frac{3}{24} \Delta t\left((1+\nu)\left|\boldsymbol{\vartheta}_{h}^{n}-\boldsymbol{y}^{n}\right|_{S}^{2}+\left(1+\nu^{-1}\right)\left|\boldsymbol{w}^{n}-\boldsymbol{y}^{n}\right|_{S}^{2}\right) \\
\leq & \alpha \frac{3}{24} \Delta t\left((1+\nu)(1+\hat{\nu})\left|\boldsymbol{\vartheta}_{h}^{n}-\boldsymbol{z}^{n}\right|_{S}^{2}+(1+\nu)\left(1+\hat{\nu}^{-1}\right)\left|\boldsymbol{z}^{n}-\boldsymbol{y}^{n}\right|_{S}^{2}\right. \\
& \left.+\left(1+\nu^{-1}\right)\left|\boldsymbol{w}^{n}-\boldsymbol{y}^{n}\right|_{S}^{2}\right) \\
\leq & \alpha \frac{3}{24} \Delta t\left((1+\nu)(1+\hat{\nu})(1+\tilde{\nu})\left|\boldsymbol{z}^{n}\right|_{S}^{2}+(1+\nu)(1+\hat{\nu})\left(1+\tilde{\nu}^{-1}\right)\left|\boldsymbol{\vartheta}_{h}^{n}\right|_{S}^{2}\right. \\
& \left.+(1+\nu)\left(1+\hat{\nu}^{-1}\right)\left|\boldsymbol{z}^{n}-\boldsymbol{y}^{n}\right|_{S}^{2}+\left(1+\nu^{-1}\right)\left|\boldsymbol{w}^{n}-\boldsymbol{y}^{n}\right|_{S}^{2}\right) \\
\leq & \alpha \frac{3}{24} \Delta t\left((1+\nu)(1+\hat{\nu})(1+\tilde{\nu})\left|\boldsymbol{z}^{n}\right|_{S}^{2}+(1+\nu)(1+\hat{\nu})\left(1+\tilde{\nu}^{-1}\right)\left|\boldsymbol{\vartheta}_{h}^{n}\right|_{S}^{2}\right) \\
& +\alpha \frac{3}{24}\left(C \Delta t h^{-1}(1+\nu)\left(1+\hat{\nu}^{-1}\right)\left\|\boldsymbol{z}^{n}-\boldsymbol{y}^{n}\right\|_{\Lambda}^{2}\right. \\
& \left.+C \Delta t h^{-1}\left(1+\nu^{-1}\right)\left\|\boldsymbol{w}^{n}-\boldsymbol{y}^{n}\right\|_{\Lambda}^{2}\right)
\end{aligned}
$$

If we choose $\nu=\frac{11+\sqrt{21}}{100}, \hat{\nu}=\frac{11-\sqrt{21}}{100}$ and $\tilde{\nu}=\frac{5}{8}$, we have

$\frac{3}{24} \Delta t\left|\boldsymbol{w}^{n}-\boldsymbol{\vartheta}_{h}^{n}\right|_{S}^{2} \leq \alpha \frac{3}{24} \Delta t\left(\beta_{\boldsymbol{z}^{n}}\left|\boldsymbol{z}^{n}\right|_{S}^{2}+\beta_{\boldsymbol{\vartheta}_{h}^{n}}\left|\boldsymbol{\vartheta}_{h}^{n}\right|_{S}^{2}\right)+\alpha \frac{3}{24}\left(\tilde{C} \Delta t h^{-1}\left\|\boldsymbol{z}^{n}-\boldsymbol{y}^{n}\right\|_{\Lambda}^{2}+\hat{C} \Delta t h^{-1}\left\|\boldsymbol{w}^{n}-\boldsymbol{y}^{n}\right\|_{\Lambda}^{2}\right)$,

with $\beta_{\boldsymbol{z}^{n}}=\frac{1599}{800}\left(\frac{3}{24} \beta_{\boldsymbol{z}^{n}}<\frac{1}{4}\right), \beta_{\boldsymbol{\vartheta}_{h}^{n}}=\frac{1599}{500}\left(\frac{3}{24} \beta_{\boldsymbol{\vartheta}_{h}^{n}}+\frac{1}{12}<\frac{1}{2}\right), \tilde{C}=C(1+\nu)\left(1+\hat{\nu}^{-1}\right)$,

$\hat{C}=C\left(1+\nu^{-1}\right)$.

This gives:

$$
\begin{aligned}
\frac{1}{2}\left\|\boldsymbol{\vartheta}_{h}^{n+1}\right\|^{2}-\frac{1}{2}\left\|\boldsymbol{\vartheta}_{h}^{n}\right\|^{2} & =-\left(\frac{5}{12}-\frac{3}{24} \beta_{\boldsymbol{\vartheta}_{h}^{n}}\right) \alpha \Delta t\left|\boldsymbol{\vartheta}_{h}^{n}\right|_{S}^{2}-\frac{1}{12} \alpha \Delta t\left|\boldsymbol{w}^{n}\right|_{S}^{2} \\
& -\frac{\alpha}{12} \Delta t\left|\boldsymbol{y}^{n}\right|_{S}^{2}-\alpha\left(\frac{1}{4}-\frac{3}{24} \beta_{\boldsymbol{z}^{n}}\right) \Delta t\left|\boldsymbol{z}^{n}\right|_{S}^{2}-\left(\frac{1}{6}-\alpha \frac{3}{24} \hat{C} \Delta t h^{-1}-\frac{1}{6} C \alpha \Delta t h^{-1}\right)\left\|\boldsymbol{y}^{n}-\boldsymbol{w}^{n}\right\|_{\Lambda}^{2} \\
& -\left(\frac{1}{4}-\alpha \frac{3}{24} \tilde{C} \Delta t h^{-1}-\frac{1}{32}\left(\Delta t C h^{-1}\right)^{2}\right)\left\|\boldsymbol{z}^{n}-\boldsymbol{y}^{n}\right\|_{\Lambda}^{2} \\
& +C \alpha \Delta t^{4} h^{-4}\left\|\boldsymbol{\vartheta}_{h}^{n}\right\|_{\Lambda}^{2} .
\end{aligned}
$$

So that under a $\frac{4}{3}$-CFL condition, we have that:

$$
\frac{1}{2}\left\|\boldsymbol{\vartheta}_{h}^{n+1}\right\|_{\Lambda}^{2}-\frac{1}{2}\left\|\boldsymbol{\vartheta}_{h}^{n}\right\|_{\Lambda}^{2} \leq C \Delta t\left\|\boldsymbol{\vartheta}_{h}^{n}\right\|_{\Lambda}^{2} .
$$

We conclude that there exists $C>0$ such that for all $n \leq M$, we find:

$$
\left\|\boldsymbol{\vartheta}_{h}^{n}\right\|_{\Lambda} \leq\left\|\boldsymbol{\vartheta}_{h}^{0}\right\|_{\Lambda} \exp (C T) .
$$

The case $\mathfrak{K}_{h} \neq 0$. If we include the contribution of the compact term, we will end up with an inequality of the same form. Indeed, there is only two types of terms. 
First the contributions of terms like $\left(\mathfrak{K}_{h}(\varphi), \varphi\right)$, with $\varphi$ that depends on $\boldsymbol{\vartheta}_{h}^{n}, \boldsymbol{w}^{n}, \boldsymbol{y}^{n}$, $z^{n}$ or a linear combination of those. Second the term coming from:

$$
-\frac{1}{24} \Delta t^{4}\left(\mathfrak{D}_{h}^{3}\left(\boldsymbol{\vartheta}_{h}^{n}\right), \mathfrak{D}_{h}\left(\boldsymbol{\vartheta}_{h}^{n}\right)\right) .
$$

Let us first note that:

$$
\begin{aligned}
\left\|\boldsymbol{w}^{n}\right\|_{\Lambda} & \leq\left\|\boldsymbol{\vartheta}_{h}^{n}\right\|_{\Lambda}+C \Delta t h^{-1}\left\|\boldsymbol{\vartheta}_{h}^{n}\right\|_{\Lambda}+C \Delta t\left\|\boldsymbol{\vartheta}_{h}^{n}\right\|_{\Lambda} \\
\left\|\boldsymbol{y}^{n}\right\|_{\Lambda} & \leq \frac{1}{2}\left\|\boldsymbol{\vartheta}_{h}^{n}\right\|_{\Lambda}+\frac{1}{2}\left\|\boldsymbol{w}^{n}\right\|_{\Lambda}+C \Delta t h^{-1}\left\|\boldsymbol{w}^{n}\right\|_{\Lambda}+C \Delta t\left\|\boldsymbol{w}^{n}\right\|_{\Lambda} \\
\left\|\boldsymbol{z}^{n}\right\|_{\Lambda} & \leq \frac{1}{3}\left\|\boldsymbol{\vartheta}_{h}^{n}\right\|_{\Lambda}+\frac{1}{3}\left\|\boldsymbol{w}^{n}\right\|_{\Lambda}+\frac{1}{3}\left\|\boldsymbol{y}^{n}\right\|_{\Lambda}+C \Delta t h^{-1}\left\|\boldsymbol{y}^{n}\right\|_{\Lambda}+C \Delta t\left\|\boldsymbol{y}^{n}\right\|_{\Lambda}
\end{aligned}
$$

This allows writing that if the $\frac{4}{3}$-CFL condition is verified and upon choosing $\Delta t$ small enough (but independently of $h$ ), there exists $C>0$, such that

$$
\begin{aligned}
\frac{1}{2} \Delta t\left(\mathfrak{K}_{h}\left(\boldsymbol{\vartheta}_{h}^{n}\right), \boldsymbol{\vartheta}_{h}^{n}\right)+\frac{1}{6} \Delta t\left(\mathfrak{K}_{h}\left(\boldsymbol{w}^{n}\right), \boldsymbol{w}^{n}\right) \\
+\frac{1}{12} \Delta t\left(\mathfrak{K}_{h}\left(\boldsymbol{y}^{n}\right), \boldsymbol{y}^{n}\right)-\frac{1}{6} \Delta t\left(\mathfrak{K}_{h}\left(\boldsymbol{w}^{n}-\boldsymbol{\vartheta}_{h}^{n}\right), \boldsymbol{w}^{n}-\boldsymbol{\vartheta}_{h}^{n}\right) \\
+\frac{1}{4} \Delta t\left(\mathfrak{K}_{h}\left(\boldsymbol{z}^{n}\right), \boldsymbol{z}^{n}\right)+\frac{1}{6}\left(-\Delta t \mathfrak{K}_{h}\left(\boldsymbol{w}^{n}-\boldsymbol{y}^{n}\right),\left(\boldsymbol{w}^{n}-\boldsymbol{y}^{n}\right)\right) \leq C \Delta t\left\|\boldsymbol{\vartheta}_{h}^{n}\right\|_{\Lambda}^{2} .
\end{aligned}
$$

For the second type of term, let us for the moment suppose that $\Lambda=I d$, to ease the reading. One has

$$
\left|\frac{1}{24} \Delta t^{4}\left(\mathfrak{D}_{h}^{3}\left(\boldsymbol{\vartheta}_{h}^{n}\right), \mathfrak{D}_{h}\left(\boldsymbol{\vartheta}_{h}^{n}\right)\right)\right|=\frac{1}{24} \Delta t^{4} \sum_{\substack{\in \in \mathbb{N}^{2}, \beta \in \mathbb{N}^{2} \\|\gamma|+|\beta|=3}}\left(\mathfrak{L}_{h}^{\gamma_{1}} \circ \mathfrak{K}_{h}^{\beta_{1}} \circ \mathfrak{L}_{h}^{\gamma_{2}} \circ \mathfrak{K}_{h}^{\beta_{2}}\left(\boldsymbol{\vartheta}_{h}^{n}\right), \mathfrak{L}_{h}+\mathfrak{K}_{h}\left(\boldsymbol{\vartheta}_{h}^{n}\right)\right) .
$$

So that

$$
\left|\frac{1}{24} \Delta t^{4}\left(\mathfrak{D}_{h}^{3}\left(\boldsymbol{\vartheta}_{h}^{n}\right), \mathfrak{D}_{h}\left(\boldsymbol{\vartheta}_{h}^{n}\right)\right)\right| \leq-\frac{1}{24} \Delta t^{4} \sum_{\gamma \in \mathbb{N}^{2}, \beta \in \mathbb{N}^{2},|\gamma|+|\beta|=3}\left(h^{-(|\gamma|+1)}+h^{-|\gamma|}\right)\left\|\boldsymbol{\vartheta}_{h}^{n}\right\|_{\Lambda}^{2} .
$$

Since $|\gamma| \leq 3$, this ensures that under the $\frac{4}{3}$-CFL condition, we have also

$$
\left|\frac{1}{24} \Delta t^{4}\left(\mathfrak{D}_{h}^{3}\left(\boldsymbol{\vartheta}_{h}^{n}\right), \mathfrak{D}_{h}\left(\boldsymbol{\vartheta}_{h}^{n}\right)\right)\right| \leq C \Delta t\left\|\boldsymbol{\vartheta}_{h}^{n}\right\|_{\Lambda}^{2} .
$$

The generalization to $\Lambda \neq I d$ but diagonal is straightforward. The result follows analogously as the case $\mathfrak{K}_{h} \equiv 0$.

Convergence of the scheme. The strategy presented here to study stability, could be used in a similar manner to prove the convergence of the scheme. However for clarity, we first wanted to present stability. For the strategy of the convergence proof, we refer the interested reader to [BEF10, LS13]. We sketch here the key ideas. Thanks to Proposition 4.1 the space-time error verifies a discrete version of the scheme with an added source term related to time consistency. The energy error principle follows from similar arguments as in the latter proof. The added terms, (due to consistency source terms) will not pose any problem for the convergence, since they do not allow for a loss of a power of $h$, so that their treatment is quite natural and do not deserve as much attention as the $\left(R_{i}\right)_{i \in[1,4]}$. Then the steps to prove convergence rely on approximation errors and similar steps as in the previous proofs. 
6. Numerical results. In this section we concentrate on the implementation and validation of the numerical approach presented here in the context of the generalized dispersive model through two test cases: the reflection spectra of a silver slab (for which some analytical reference solutions are available) and the computation of the cross section for a gold nanoshell. This has been implemented in the frame of a MPI-parallel Fortran 90 Discontinuous Galerkin code.

6.1. Validation. To validate our implementation of the generalized dispersive model in the DGTD framework, we consider a simple setup composed of a doubly periodic silver slab of thickness $10 \mathrm{~nm}$. The latter is illuminated by a wideband plane wave in normal incidence. In this particular case, we have access to an analytical expression of the reflection coefficient for each frequency of the incident wave (see LSV13, for details). As in section 2, we are interested in the $[300,1500] \mathrm{THz}$ frequency range. We describe the gold by a 4SOGP model, which parameters are available in [LSV13]. We compute the solution with a $\mathbb{P}_{4}$ polynomial approximation on a simplicial mesh. For a sufficiently long simulation (and physical) time, the Fourier transform of the scattered field is recorded on-the-fly at a probe point located before the slab. At the end of the numerical computation, a numerical reflection coefficient is computed and compared with the analytical one. The setup is presented on figure 5(a), while the results are displayed in figure 5(b). For this model as well as for other test problems not discussed in this paper, we obtain a perfect agreement between analytical and computed solution, which validates our implementation of the Generalized Dispersive model in the DGTD framework. We furthermore focused on a upwind scheme (i.e. $\alpha=1$ ). We also investigated the interest of choosing a generalized dispersive

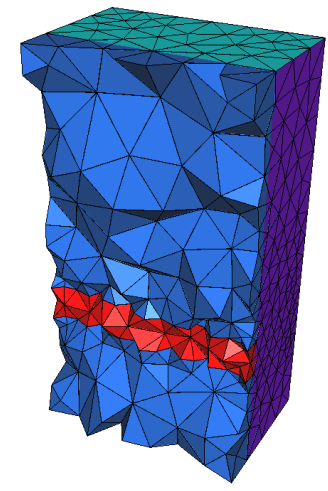

(a) Mesh of the doubly periodic silver slab. The slab is in red, while the blue corresponds to vacuum. Purple boundary triangles correspond to periodic conditions, while on green ones an absorbing boundary condition is imposed.

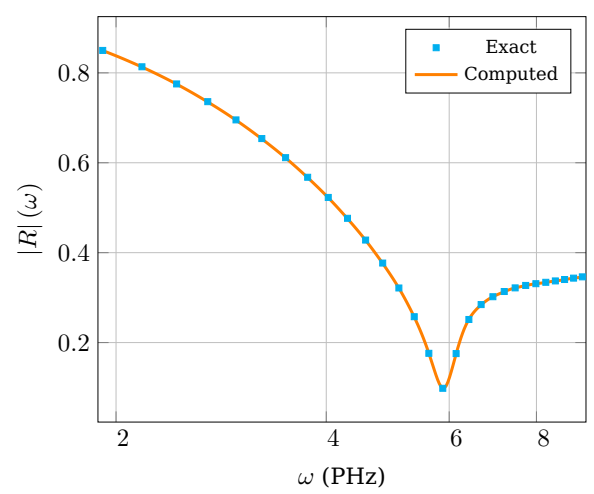

(b) Exact and computed reflection spectra for a 4SOGP fit. As can be seen the DGTD results are in excellent agreement with the analytical solution.

FIG. 5. Set-up (5(a) and results $5(5)$ of the doubly periodic silver slab. $\mathbb{P}_{4}$ polynomial approximation is used for the spatial DG approximation.

model : on figure 6, the plots of silver imaginary part permittivity and the predicted reflectance spectra are presented aside for the experimental data of [JC72], and the 2SOGP and 4SOGP models. The positive impact of a good fitting of the material properties is particularly visible here at the resonance frequency, since the relative 
error on the amplitude of the resonance drops from $90 \%$ for 2SOGP fitting to roughly $33 \%$ for 4 SOGP for an extra computational time of $5 \%$ only.

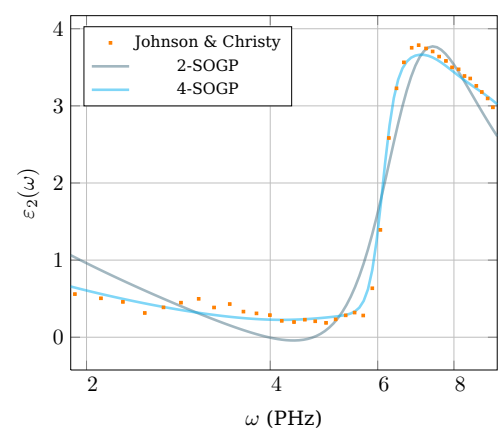

(a) Permittivity (imaginary part)

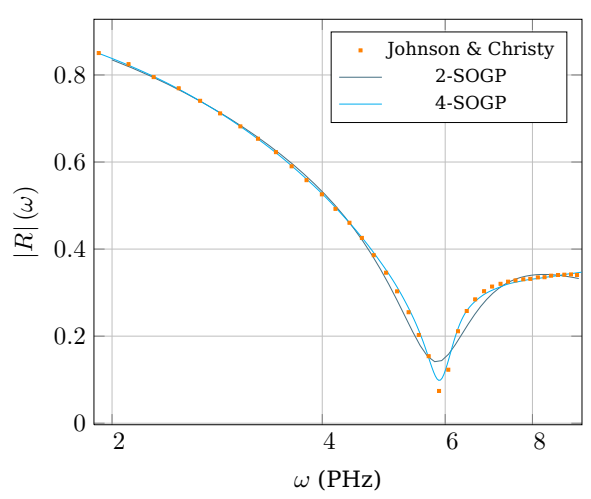

(b) Computed reflection spectra

FIG. 6. Comparison of the fitting of imaginary part of the permittivity silver by 2-SOGP and 4-SOGP and its impact on the prediction of the reflectance spectrum.

6.2. Absorption cross-section of a gold nanoshell. In this section, we are concerned with the study if the illumination of a core-silica-gold-shell device, for which $R_{\text {in }}=150 \mathrm{~nm}$, and $R_{\text {out }}=172 \mathrm{~nm}$. To describe its reaction we compute its absorption cross-section. The latter is quantifying the absorption response of the nanoshell in the considered frequency range via the computation of a normalized scattering Poynting vector flux on a given surface (see BKN11 for more details). To compute this quantity we used a Total-Field/Scattered-Field (TF/SF) formulation and enclose the scatterer sphere in the TF/SF interface on which the scattering cross-section will be computed (see BKN11) . The Fourier transforms of the $\mathbf{E}$ and $\mathbf{H}$ fields are computed on-thefly on the TF/SF interface, and the absorption cross-section is eventually computed at the end of the simulation. The reader is referred to [BKN11 for details about this process. We consider increasingly-complex gold dispersion models (in terms of poles in the generalized model) to describe the shell. The silica core is described by a constant $\varepsilon=1.5$ permittivity. We selected and present the results for Drude and 4SOGP model in figure 7. As could be expected from what was presented in section 2, the high-frequency behavior of the scatterer is strongly modified when an enhanced dispersion model is used. Hence, depending on the considered frequency range, a careful selection of the number of poles should be done, 4 second-order generalized poles being a good compromise for wide-band computations. To support this statement, the induced overhead was calculated for different dispersion models. In average, a dispersive tetrahedron only requires $4.6 \%$ additional memory space and $0.6 \%$ additional CPU time per pole compared to a non-dispersive one. Given that the amount of dispersive tetrahedra in classical nano-optics devices is usually less than $20 \%$ of the total, this makes the generalized dispersive model a cheap way to achieve a good description of the material properties.

7. Conclusion. In this paper, we have presented a complete study of several dispersive models used in the context of nanophotonics via a generalized dispersive model. Starting from the theoretical point of view of existence and uniqueness of a solution, we went through the study of Discontinuous Galerkin Time Domain semi- 


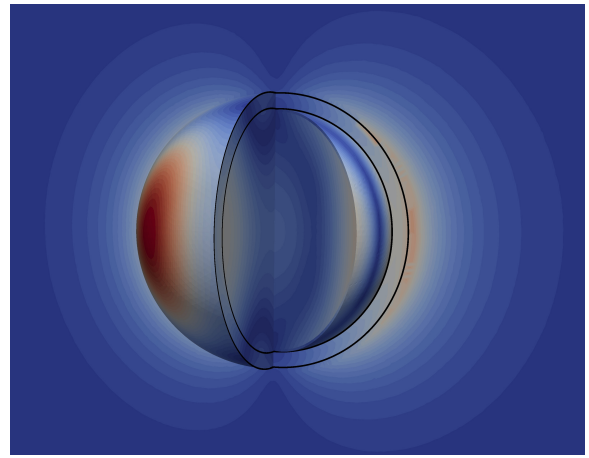

(a) Modulus of the $\mathbf{E}$ field in the vicinity of the nanoshell at $t=2 \times 10^{-14} \mathrm{~s}$. in the $x-y$ plane. A 4SOGP dispersion model was used to describe the gold shell.

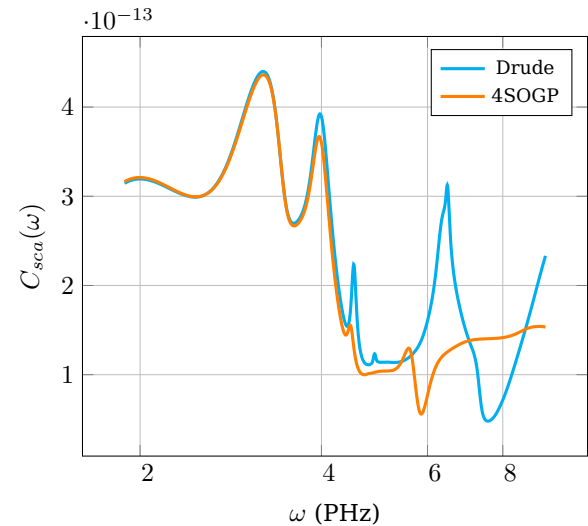

(b) Computed scattering cross-sections of the nanoshell for various gold dispersion models. The dispersion parameters can be found in LSV13.

FIG. 7. E near-field solution (7(a) $)$ and scattering cross-section $(7(b))$ of the silica/gold nanoshell device. $\mathbb{P}_{4}$ polynomial approximation is used for the spatial DG approximation, along with curvilinear elements for an enhanced geometrical description of the shell.

and fully-discrete schemes and proceeded with realistic nanophotonics test cases. The generalized dispersive model notably improves the quality of the solution over classical models for a constant number of poles. It also proved to be very efficient in the specific case of transition metals. The significance of an enhanced description of the metal dispersion has been underlined, while the induced overhead has been shown to be very reasonable.

Future developments will be mainly concerned with the modeling of more complex dispersive phenomena, such as non-local effects MCS13. The latter occur in dispersive structures including small geometrical structures or gaps (typically smaller than $10 \mathrm{~nm}$ ), giving rise to bulk plasmon excitations. Numerical investigations on this model were conducted in 2D (see $\left[\mathrm{SSL}^{+} 15\right)$, and will soon be extended to 3D.

\section{REFERENCES}

[AH03] H. Ammari and K. Hamdache. Global existence and regularity of solutions to a system of nonlinear Maxwell's equations. J. Math. Anal. Appl., 286:51-63, 2003.

[BEF10] E. Burman, A. Ern, and M. A. Fernandez. Explicit Runge Kutta schemes and finite elements with symmetric stabilization for first-order linear PDE systems. SIAM J. Numer. Anal., 48(6):2019-2042, 2010.

[BKN11] K. Busch, M. König, and J. Niegemann. Discontinuous Galerkin methods in nanophotonics. Laser and Photonics Reviews, 5:1-37, 2011.

[CC41] K. S. Cole and R. H. Cole. Dispersion and absorption in dielectrics - I Alternating current characteristics. Journal of Chemical Physics, 9:341-352, 1941.

[CH90] T. Cazenave and A. Haraux. Introduction aux problèmes d'évolution semi-linéaires. Mathematics and Applications. Ellipses, 1990.

[Dru00] P. Drude. Zur elektronentheorie der metalle. Annalen der Physik, 306:566-613, 1900.

[Fle78] J. W. Fleming. Material dispersion in lightguide glasses. Electronics Letters, 14:326328,1978 .

[FLLP05] L. Fezoui, S. Lanteri, S. Lohrengel, and S. Piperno. Convergence and stability of a discontinuous Galerkin time-domain method for the 3D heterogeneous Maxwell equations on unstructured meshes. ESAIM Math. Model. Num. Anal. (M2AN), 
39(6):1149-1176, 2005.

[GGC96] C. Gabriel, S. Gabriel, and E. Corthout. The dielectric properties of biological tissues: I. Literature survey. Physics in Medicine and Biology, 41:2231-2249, 1996.

[GS99] B. Gustavsen and S. Semlyen. Rational approximation of frequency domain responses by vector fitting. Trans. Power Del., 14:1052-1061, 1999.

[GYKR12] S. D. Gedney, J. C. Young, T. C. Kramer, and J. A. Roden. A discontinuous Galerkin finite element time-domain method modeling of dispersive media. IEEE Transactions on Antennas and Propagation, 60:1969-1977, 2012.

[HDF06] M. Han, R.W. Dutton, and S. Fan. Model dispersive media in FDTD method with complex-conjudate pole-residue pairs. Trans. Microw. Wireless Compon. Lett., 16:119-121, 2006.

[HN07] F. Hao and P. Nordlander. Efficient dielectric function for FDTD simulation of the optical properties of silver and gold nanoparticles. Chemical Physics Letters, 446:115-118, 2007.

[HP15] M. Hochbruck and T. Pazur. Implicit runge-kutta methods and discontinuous Galerkin discretizations for linear Maxwell's equations. SIAM J. Numer. Anal., 53(1):485$507,2015$.

[JC72] P.B. Johnson and R.W. Christy. Optical constants of the noble metals. Physical Review B, 6:4370-4379, 1972.

[JC74] P.B. Johnson and R.W. Christy. Optical constants of transition metals: Ti, V, Cr, Mn, Fe, Co, Ni, and Pd. Physical Review B, 9:5056-5070, 1974.

[JL06] y. Chen J. Li. Analysis of a time domain finite element method for 3d maxwell's equations in dispersive media. Comput. Meth. App. Mech. Engng., 195:42204229, 2006.

[KGV83] S. Kirkpatrick, C.D. Gelatt, and M.P. Vecchi. Optimization by simulated annealing. Science, 220:671-680, 1983.

[LC09] J. Y. Lu and Y. H. Chang. Implementation of an efficient dielectric function into the finite difference time domain method for simulating the coupling between localized surface plasmons of nanostructures. Superlattices and Microstructures, 47:60-65, 2009.

[LCE08] J. Li, Y. Chen, and V. Elander. Mathematical and numerical study of wave propagation in negative-index material. Comput. Meth. App. Mech. Engng., 197:3976-3987, 2008.

[Li07] J. Li. Error analysis of fully discrete mixed finite element schemes for 3-D Maxwell's equations in dispersive media. Comput. Meth. App. Mech. Engng., 196:3081-3094, 2007.

[Li09] J. Li. Numerical convergence and physical fidelity analysis for Maxwell's equations in metamaterials. Comput. Meth. App. Mech. Engng., 198:3161-3172, 2009.

[LS13] S. Lanteri and C. Scheid. Convergence of a discontinuous Galerkin scheme for the mixed time domain Maxwell's equations in dispersive media. IMA J. Numer. Anal., 33(2):432-459, 2013.

[LSV13] S. Lanteri, C. Scheid, and J. Viquerat. Theoretical and numerical analysis of local dispersion models coupled to a discontinuous Galerkin time-domain for Maxwell's equations, rr-8298. Technical report, Inria Sophia Antipolis, Project-team Nachos, 2013.

[LT98] D. Levy and E. Tadmor. From semidiscrete to fully discrete: stability of runge-kutta schemes by the energy method. SIAM Rev., 40:40-73, 1998.

[MCS13] A. Moreau, C. Ciraci, and D. R. Smith. The impact of nonlocal response on metallodielectric multilayers and optical patch antennas. Physical Review B, 87, 2013.

[NH07] B. Novotny and L. Hecht. Principles of nano-optics. Cambridge University Press, first edition, 2007.

[Pal98] E. D. Palik. Handbook of Optical Constants of Solids. Academic Press, 1998.

[Paz83] A. Pazy. Semigroups of linear operators and applications to partial differential equations, volume 44 of Applied Mathematical Sciences. Springer-Verlag, 1983.

$\left[\mathrm{PPM}^{+}{ }_{14} \quad\right.$ Z. Pirzadeh, T. Pakizeh, V. Miljkovic, C.Langhammer, and A. Dmitriev. Plasmoninterband coupling in Nickel nanoantennas. ACS Photonics, 1:158-162, 2014.

$\left[\mathrm{SSL}^{+}{ }^{15}\right] \quad$ N. Schmitt, C. Scheid, S. Lanteri, J. Viquerat, and A. Moreau. A dgtd method for the numerical modeling of the interaction of light with nanometer scale metallic structures taking into account non-local dispersion effects, RR-8726, pp.73. Technical report, Inria Sophia Antipolis, Project-team Nachos, 2015.

[Tit48] E. C. Titchmarsh. Introduction to the Theory of Fourier Integrals. Oxford at the Clarendon Press, 1948. 
[VLDC11] A. Vial, T. Laroche, M. Dridi, and L. Le Cunff. A new model of dispersion for metals leading to a more accurate modeling of plasmonic structures using the FDTD method. Applied Physics A, 103:849-853, 2011.

[WROB13] C. Wolff, R. Rodriguez-Oliveros, and K. Busch. Simple magneto-optic transition metal models for time-domain simulations. Optics Express, 21:12022-12037, 2013.

[WXZ10] B. Wang, Z. Xie, and Z. Zhang. Error analysis of a discontinuous Galerkin method for Maxwell equations in dispersive media. J. Comput. Phys, 229:8552-8563, 2010. 